ARTICLE

\title{
Intracellular trafficking of Notch orchestrates temporal dynamics of Notch activity in the fly brain
}

\author{
Miaoxing Wang (10 1,6, Xujun Han (1) 1,6, Chuyan Liu (10 2 ${ }^{2}$, Rie Takayama1', Tetsuo Yasugi ${ }^{1}$, Shin-Ichiro Ei ${ }^{3}$,

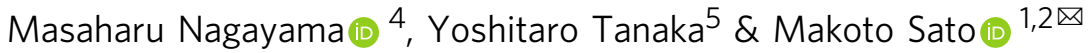

While Delta non-autonomously activates Notch in neighboring cells, it autonomously inactivates Notch through cis-inhibition, the molecular mechanism and biological roles of which remain elusive. The wave of differentiation in the Drosophila brain, the 'proneural wave', is an excellent model for studying Notch signaling in vivo. Here, we show that strong nonlinearity in cis-inhibition reproduces the second peak of Notch activity behind the proneural wave in silico. Based on this, we demonstrate that Delta expression induces a quick degradation of Notch in late endosomes and the formation of the twin peaks of Notch activity in vivo. Indeed, the amount of Notch is upregulated and the twin peaks are fused forming a single peak when the function of Delta or late endosomes is compromised. Additionally, we show that the second Notch peak behind the wavefront controls neurogenesis. Thus, intracellular trafficking of Notch orchestrates the temporal dynamics of Notch activity and the temporal patterning of neurogenesis.

\footnotetext{
${ }^{1}$ Mathematical Neuroscience Unit, Institute for Frontier Science Initiative, Kanazawa University, Kanazawa, Ishikawa, Japan. ${ }^{2}$ Laboratory of Developmental Neurobiology, Graduate School of Medical Sciences, Kanazawa University, Kanazawa, Ishikawa, Japan. ${ }^{3}$ Department of Mathematics, Faculty of Science, Hokkaido University, Sapporo, Hokkaido, Japan. ${ }^{4}$ Research Institute for Electronic Science, Research Center of Mathematics for Social Creativity, Hokkaido University, Sapporo, Hokkaido, Japan. ${ }^{5}$ Department of Complex and Intelligent Systems, School of Systems Information Science, Future University Hakodate, Hakodate, Hokkaido, Japan. ${ }^{6}$ These authors contributed equally: Miaoxing Wang, Xujun Han. ${ }^{凶}$ email: makotos@staff.kanazawa-u.ac.jp
} 
$\mathrm{N}$ otch $(\mathrm{N})$ signaling plays diverse roles in many biological processes $^{1}$. N-mediated lateral inhibition is reiteratively used to select a small number of differentiated cells from a large number of undifferentiated cells in a spatially and temporally regulated manner ${ }^{2}$. We previously demonstrated that $\mathrm{N}$ mediated lateral inhibition regulates the speed of proneural wave progression when combined with epidermal growth factor (EGF)mediated reaction diffusion ${ }^{3,4}$.

A membrane-bound ligand, Delta (Dl), plays major roles in $\mathrm{N}$ mediated lateral inhibition ${ }^{5}$. It non-autonomously activates $\mathrm{N}$ in adjacent cells through a process "trans-activation." Upon binding with $\mathrm{Dl}$, the intracellular domain of $\mathrm{N}$ is cleaved to produce the $\mathrm{N}$ intracellular domain $\left(\mathrm{N}^{\mathrm{ICD}}\right)$, which forms a complex with a DNA-binding transcription regulator, Suppressor of Hairless (Su $(\mathrm{H})$ ), and regulates target gene transcription ${ }^{6-8}$. On the other hand, $\mathrm{N}$ is autonomously inactivated by $\mathrm{Dl}$ expressed in the same cell through a process "cis-inhibition," whose molecular mechanism and biological significance remain largely elusive ${ }^{9-12}$.

The direct interaction between $\mathrm{Dl}$ and $\mathrm{N}$ seems to trigger cisinhibition by inhibiting $\mathrm{N}$ prior to or following its transport to the plasma membrane ${ }^{13}$. There are two possible mechanisms of cisinhibition. First, the cis-interaction of the ligand and receptor may shut off the transport of $\mathrm{N}$ from the endoplasmic reticulum (ER) to the plasma membrane ${ }^{14}$. Second, the cis-interaction may trigger the catalytic process that results in $\mathrm{N}$ degradation. For example, the $\mathrm{Dl}-\mathrm{N}$ complex may be internalized from the plasma membrane to cause $\mathrm{N}$ degradation. Protein degradation in late endosomes has been shown to play important roles in activating and inactivating $\mathrm{N}$ signaling during trans-activation ${ }^{15-24}$. However, the potential roles of intracellular trafficking of $\mathrm{Dl}$ and $\mathrm{N}$ in cis-inhibition remain largely unknown.

On the surface of the developing fly brain, the wave of differentiation, "proneural wave," propagates along the twodimensional sheet of neuroepithelial cells (NEs), which sequentially differentiate into neuroblasts (NBs), the neural stem-like cells. In the previous study, we formulated a mathematical model of the proneural wave, which includes $\mathrm{N}$ activity $(N)$, Dl expression $(D)$, EGF signal activity $(E)$, and the state of NB differentiation $(A)$. $A$ is related to the expression levels of Achaete-Scute Complex proteins (AS-C). The model successfully reproduces the complex behaviors of the proneural wave in various genetic backgrounds ${ }^{25,26} . \mathrm{N}$ is activated along the wavefront, forming an activity peak that negatively regulates the wave propagation ${ }^{27,28}$. However, $\mathrm{N}$ is activated again behind the proneural wave, showing twin peaks of $\mathrm{N}$ activity in vivo ${ }^{3,29}$ (Fig. 1a-d). If the location of Dl-expressing cells does not change, the combination of trans-activation and cis-inhibition would robustly form the twin activity peaks of Notch ${ }^{11}$. However, Dl expression propagates as the proneural wave progresses. The mechanism that forms the twin peaks of $\mathrm{N}$ activity and the biological significance of the second $\mathrm{N}$ activity peak have not been addressed thus far.

Behind the proneural wave, NBs start producing diverse types of neurons. The production of neural diversity is controlled by the transition of temporal transcription factors sequentially expressed in NBs. Homothorax (Hth), Klumpfuss (Klu), Eyeless (Ey), Sloppy paired (Slp), Dichaete (D), and Tailless (Tll) are known to act as the temporal transcription factors in the developing medulla (Fig. 1b) ${ }^{30,31}$. While Hth expression is already upregulated in NEs prior to NB differentiation, the expression of the other temporal factors is upregulated behind the proneural wave. Thus, the proneural wave could be the initial trigger of the temporal transcription factor cascade following Klu. Since the second $\mathrm{N}$ peak is found in $\mathrm{NBs}$ behind the wavefront, $\mathrm{N}$ signaling could trigger the transition of temporal transcription factor expression.
In this study, we reproduce the twin peaks of $\mathrm{N}$ activity by modifying the previous mathematical model and demonstrate that a strong nonlinearity in cis-inhibition robustly reproduces the twin peaks. As a potential candidate mechanism of the nonlinear behavior of cis-inhibition, we assume that $\mathrm{Dl}$ may transport $\mathrm{N}$ to late endosomes, in which Rab7 and the ESCRT (endosomal sorting complexes required for transport) complex quickly degrade $\mathrm{N}$, which results in the inactivation of $\mathrm{N}$ between the twin peaks. Indeed, partial knockdown of $D l$ or inactivation of ESCRT complex causes upregulation of $\mathrm{N}$ activity and fusion of the twin peaks, forming a single peak of $\mathrm{N}$ activity. These results support the idea that intracellular trafficking of $\mathrm{N}$ triggers cisinhibition, which changes the dynamics of $\mathrm{N}$ activity. We further explore the biological significance of the twin peaks of $\mathrm{N}$ activity. Interestingly, the second $\mathrm{N}$ activity peak coincides with Klu expression in the medulla NBs. We demonstrate that Klu expression depends on $\mathrm{Dl}$ expression along the proneural wavefront, and the formation of the single $\mathrm{N}$ peak by inhibiting ESCRT function results in the abnormal temporal patterning of NBs and neurons. Thus, we demonstrate the molecular mechanism of cis-inhibition that orchestrates the temporal dynamics of $\mathrm{N}$ activity and the temporal patterning of neurogenesis.

\section{Results}

Nonlinear cis-inhibition establishes the twin peaks. In our previous mathematical model, the cis-inhibition term was proportional to $\mathrm{Dl}$ expression ${ }^{3}$. When the magnitude of trans-activation and cis-inhibition is roughly equivalent, $\mathrm{N}$ is activated only once around the wavefront. However, $\mathrm{N}$ is activated again behind the wavefront, showing the twin activity peaks (Fig. 1a-d) ${ }^{3,29}$. We used two independent $\mathrm{N}$ activity markers. $\mathrm{E}(\mathrm{spl}) \mathrm{m} \gamma$-GFP (m $\gamma$ GFP) shows nuclear signals that are more prominent in the first peak than in the second peak (Fig. 1d) ${ }^{32}$. In contrast, NREdVenus shows cytoplasmic signals that are more prominent in the second peak than in the other (Fig. 1c) ${ }^{33}$.

If Dl is continuously expressed in the same cells, the combination of trans-activation and cis-inhibition would robustly form the twin peaks of Notch activity ${ }^{11}$. However, Dl-expressing cells change as the proneural wave progresses. To generate a robust gap between the twin peaks, $\mathrm{N}$ must be quickly inactivated just after the first activity peak. Since $\mathrm{Dl}$ is specifically expressed at the wavefront (Fig. 1b), Dl-mediated cis-inhibition could be the cause of the inactivation following the first peak and the formation of the twin peaks of $\mathrm{N}$ activity.

We asked if the twin peaks could be reproduced by modifying cis-inhibition in the mathematical model of the proneural wave. It was demonstrated that the kinetics of cis-inhibition are very fast compared with the gradual effect of trans-activation from a series of in vitro experiments ${ }^{11}$. Thus, we incorporated a step function term for cis-inhibition, with which cis-inhibition does not occur when $\mathrm{Dl}$ expression is below the threshold $\left(e_{c}\right)$, but quickly inactivates $\mathrm{N}$ when $\mathrm{Dl}$ expression exceeds the threshold (Fig. 1f). The twin peaks pattern of $\mathrm{N}$ activity was reproduced without significantly compromising the magnitude of $\mathrm{N}$ activity for a wide variety of parameter settings (Fig. 1e, f and Supplementary Figure 1).

However, the step function is very artificial and nonbiological. According to the previous literature ${ }^{11}$, we incorporated the Hill functions for trans-activation and cis-inhibition. The Hill function is commonly used to model a biochemical reaction, in which the Hill's coefficient $\left(n_{\mathrm{t}}\right.$ and $\left.n_{\mathrm{c}}\right)$ and activation coefficient $\left(k_{\mathrm{t}}\right.$ and $k_{\mathrm{c}}$; Fig. $1 \mathrm{~g}$ ) specifies the kinetics of the reaction. We systematically modified the Hill's and activation coefficients of $c i s$-inhibition to modify its response speed (Fig. $1 \mathrm{~g}$ 

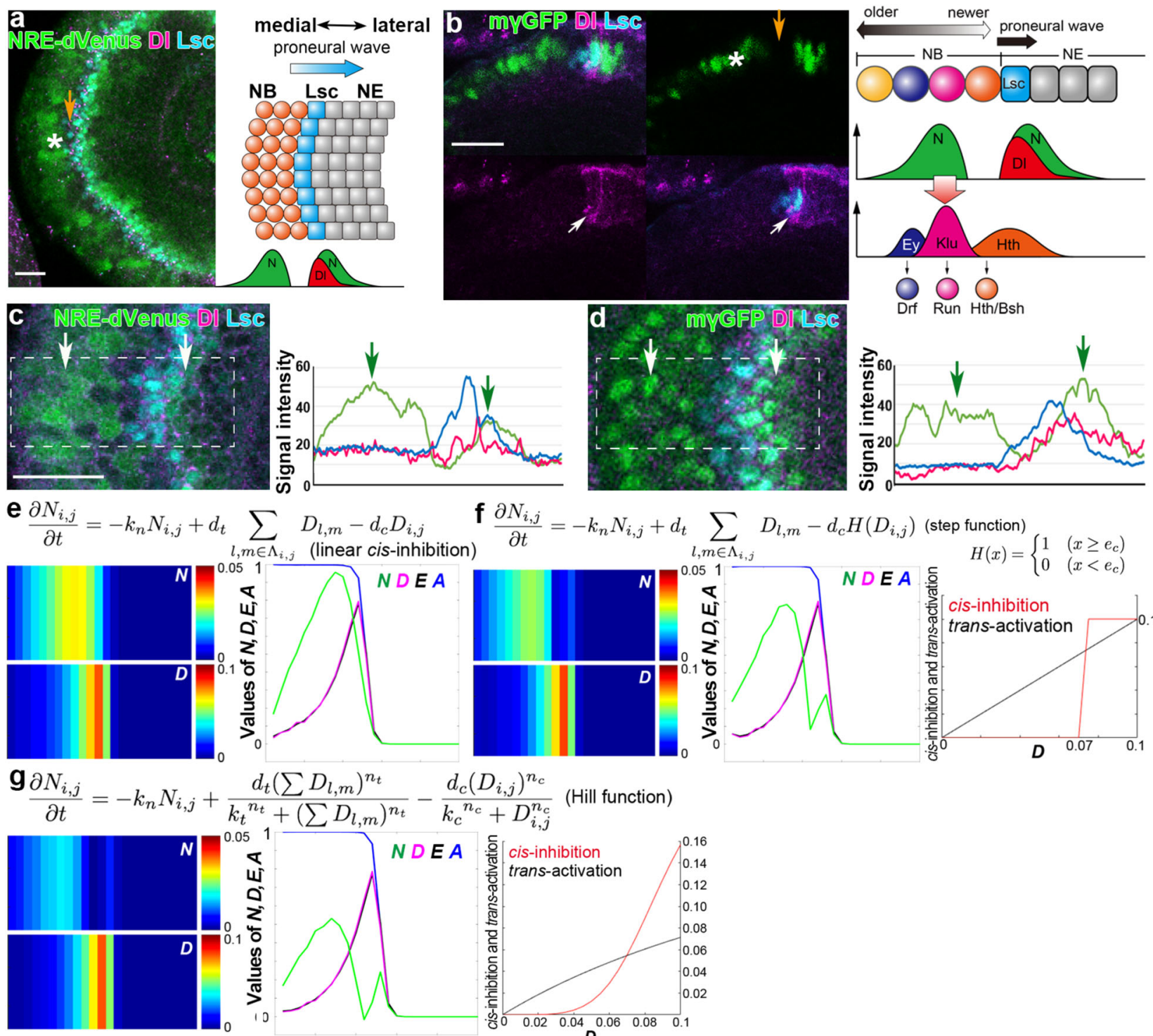

$$
\frac{d_{c}\left(D_{i, j}\right)^{n_{c}}}{n_{c}^{n_{c}}+D_{i, j}^{n_{c}}}
$$

(Hill function)

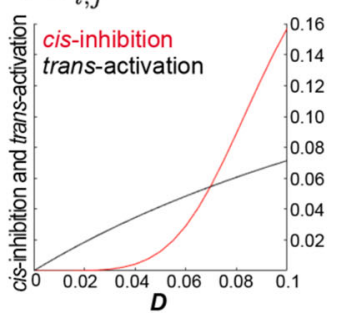

Fig. 1 Nonlinear cis-inhibition establishes the twin peaks of Notch activity. a Optic lobe in a lateral view showing the twin peaks of Notch activity (NREdVenus, green), wavefront (Lsc, blue), and DI (magenta) in the left panel. The proneural wave progresses in a medial-to-lateral orientation (left to right). Asterisk indicates the second peak of $\mathrm{N}$ activity in NBs. Orange arrow indicates the gap between the twin peaks. The right panel shows that Lsc expression at the wavefront triggers the differentiation from NE to NB and overlaps with DI expression and the first peak of $\mathrm{N}$ activity. $\mathbf{b} \mathrm{A}$ section showing the twin peaks of Notch activity (myGFP, green), wavefront (Lsc, blue), and DI (magenta). White arrows show DI expression at the wavefront. Orange arrow indicates the gap between the twin peaks of $\mathrm{N}$ activity. Schematic shows that NBs sequentially express temporal transcription factors, Hth (orange), Klu (magenta), and Ey (blue), which control the production of neurons expressing Hth/Bsh, Run, and Drf, respectively. c, d N activity is visualized by NRE$\mathrm{dVenus}(\mathbf{c})$ and m $\gamma$ GFP (d) in green and compared with the distributions of DI (magenta) and Lsc (blue). Signals in the dotted box areas are plotted in the right panels. Arrows indicate the twin peaks of $\mathrm{N}$ activity. Scale bars indicate $20 \mu \mathrm{m}$ (a-d). e-g Results of numerical simulations: left panels show the twodimensional patterns of $N$ and $D$. Middle panels show the one-dimensional patterns of $N$ (green), $D$ (magenta), $E$ (black), and $A$ (blue; $y=13$ ). The values of $N, D$, and $E$ are 30,10 , and 10 times multiplied, respectively. Right panels in (f, $\mathbf{g}$ ) compare the profiles of trans-activation (black) and cis-inhibition (red) in response to $D$. The values of trans-activation are four times multiplied because one cell could receive trans-activation from as many as four adjacent cells. e Single peak of $\mathrm{N}$ activity in the previous mathematical model in which the effect of cis-inhibition is linear. $\mathbf{f}, \mathbf{g}$ Twin peaks of $\mathrm{N}$ activity are reproduced by introducing step function (f) or Hill function $(\mathbf{g})$ to cis-inhibition.

and Supplementary Figure 2). The twin peaks pattern of $\mathrm{N}$ activity, which is similar to the in vivo pattern (Fig. 1a, c, d), was reproduced when the kinetics of cis-inhibition is faster compared with that of trans-activation.

Delta activates Notch activity behind the wavefront. We asked if the mechanism demonstrated in the above in silico experiments exists in vivo. As already demonstrated, the $\mathrm{N}$ signal is activated in cells adjacent to a clone of cells ectopically expressing $\mathrm{Dl}$, while it is efficiently inactivated in NE cells expressing Dl (Fig. 2a) $)^{13,29}$ Since Dl-expressing cells also receive trans-activation from surrounding Dl-expressing cells, cis-inhibition appears to be dominant over trans-activation, which is consistent with the idea that the kinetics of cis-inhibition are faster compared with that of trans-activation.

To address this idea in a loss-of-function condition, we utilized the mathematical model. If the strong expression level of $\mathrm{Dl}$ at the wavefront is the trigger of cis-inhibition, a mild reduction in $\mathrm{Dl}$ 

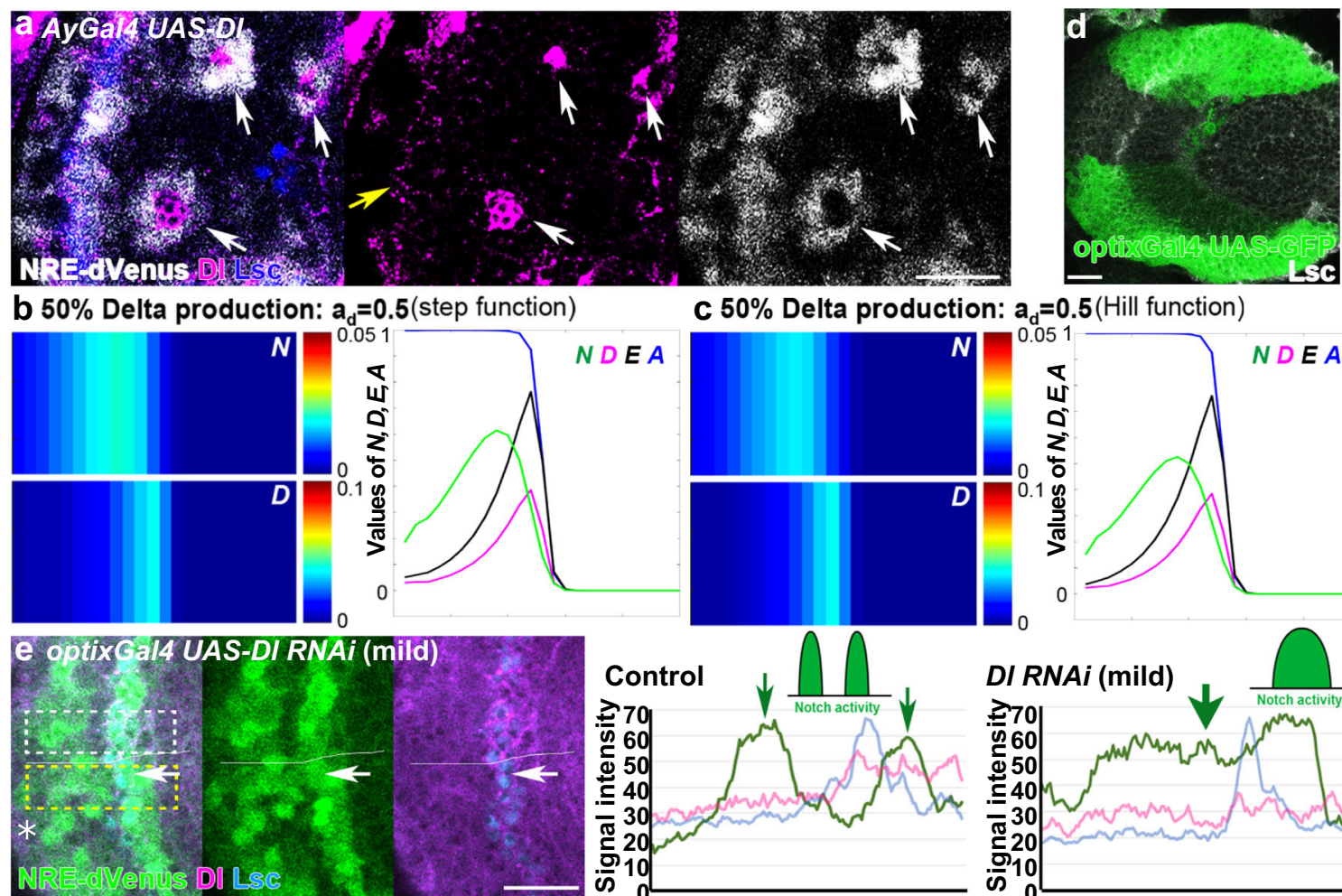

C $\mathbf{5 0 \%}$ Delta production: $\mathbf{a}_{\mathrm{d}}=\mathbf{0 . 5}$ (Hill function)
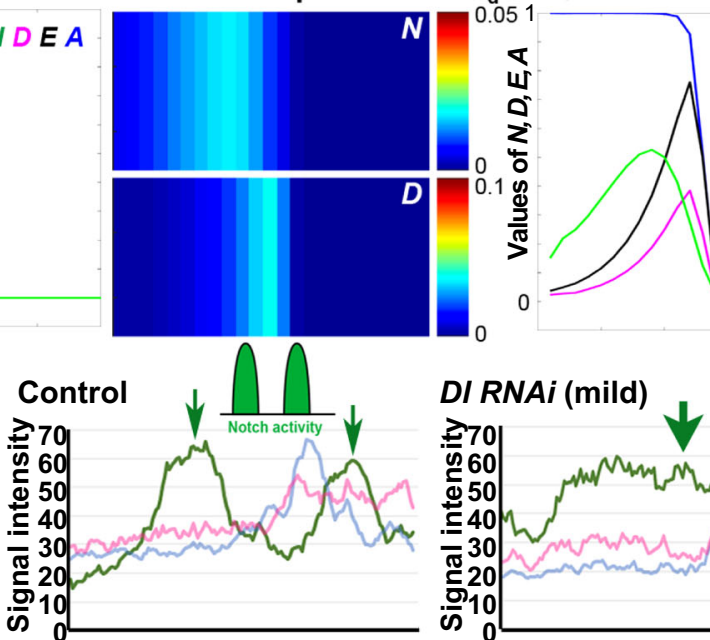

NDEA
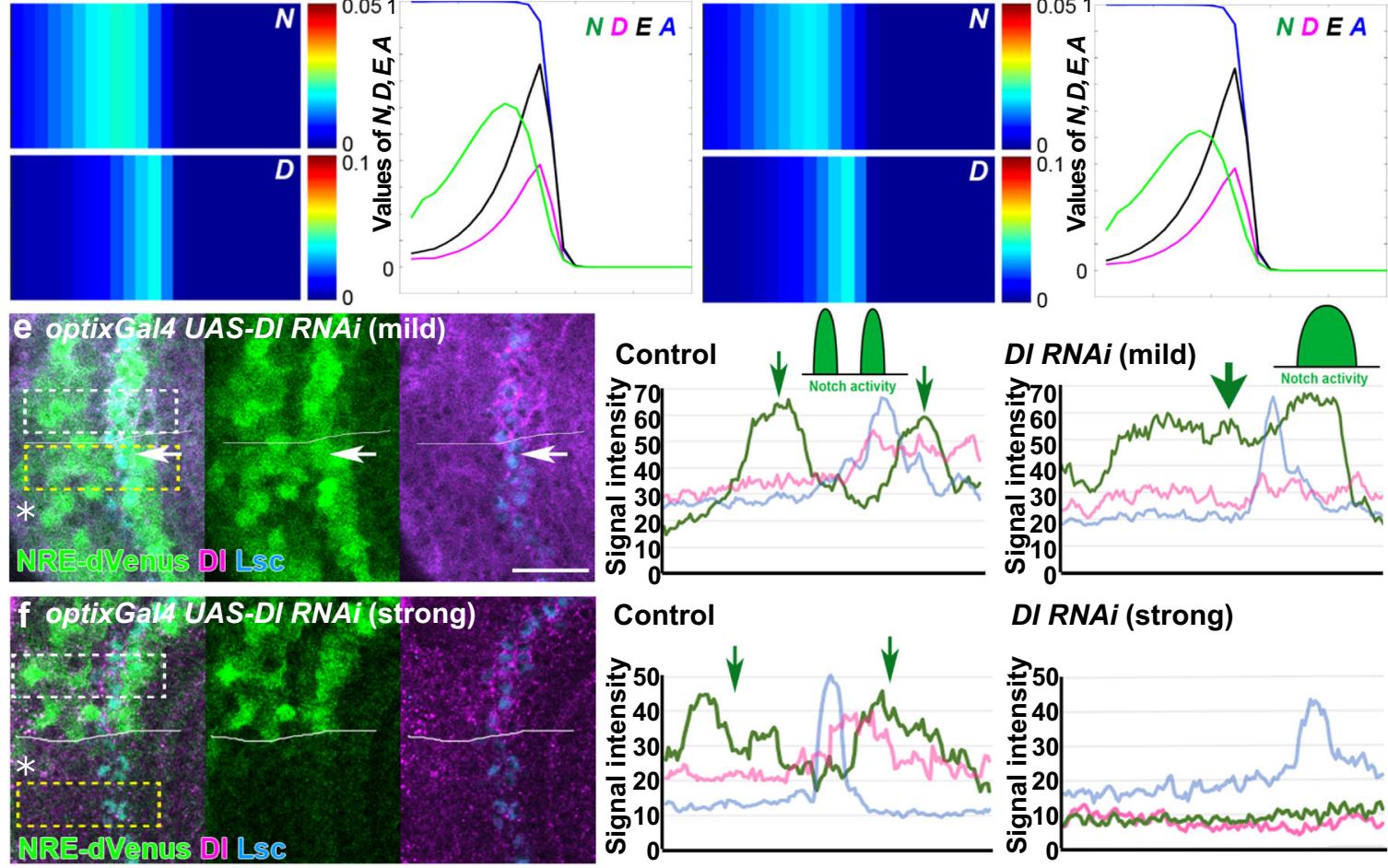

DI RNAi (strong)
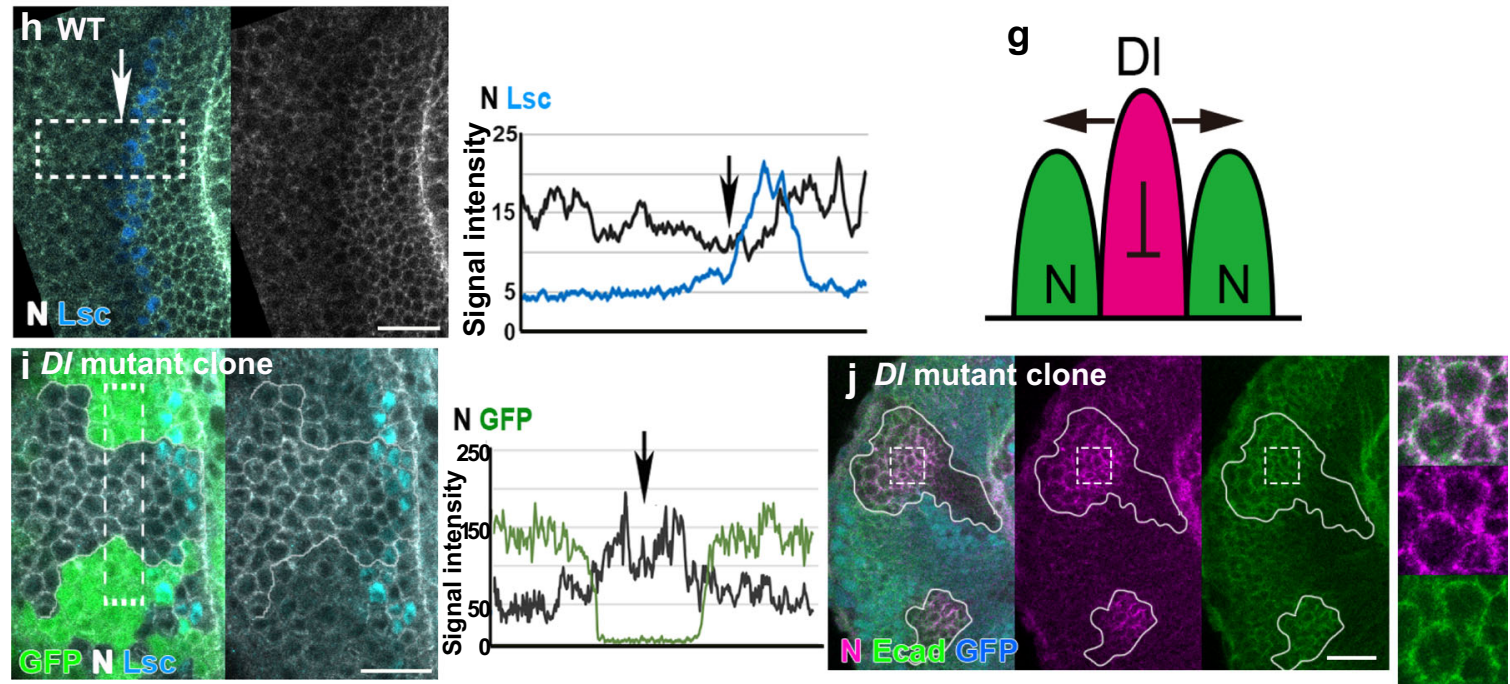

production should cause the fusion of the twin peaks, forming a single $\mathrm{N}$ activity peak (Fig. $2 \mathrm{~b}, \mathrm{c}$ ). To test this idea in vivo, we made use of two different UAS-Dl RNA interference (RNAi) strains. When expressed in the retina under the control of GMRGal4, the mild $D l$ RNAi reduced the eye width from 0.37 to 0.35 $\mathrm{mm}$, while it was further reduced to $0.31 \mathrm{~mm}$ by the strong $D l$ RNAi (Supplementary Figure 3).

We found that the mild $D l$ RNAi under the control of optixGal4, which is expressed in the dorsal and ventral subdomains of the optic lobe (Fig. 2d), causes the single $\mathrm{N}$ peak. The twin $\mathrm{N}$ activity peaks were fused to become a single peak (Fig. 2e and Supplementary Figure 4a). When we removed Dl expression by expressing the strong RNAi strain, both of the twin peaks of $\mathrm{N}$ activity were eliminated (Fig. 2f and Supplementary Figure 4b) and the same result was also observed in the $D l$ mutant clone (Supplementary Figure 4c). Note that $\mathrm{Dl}$ is specifically expressed at the proneural wavefront cells (Fig. 1b), suggesting that this Dl expression is responsible for the twin activation of $\mathrm{N}$ signaling in 
Fig. 2 Delta represses Notch activity at the wavefront. a N activity (NRE-dVenus, white) is elevated in cells adjacent to DI-expressing clones (Ay-Gal4 UAS-DI, magenta), and is autonomously repressed (arrows). Yellow arrow indicates endogenous DI expression at the wavefront (Lsc, blue). White and magenta signals are enhanced. b, c Results of numerical simulations using step function (b) and Hill function (c). Left panels show the two-dimensional patterns of $N$ and $D$. Right panels are the one-dimensional plots of $N$ (green), D (magenta), $E$ (black), and $A$ (blue; $y=13$ ). The values of $N, D$, and $E$ are 30 , 10 , and 10 times multiplied, respectively. Reduced production rate of $D$ causes single peak of $N$ activity $\left(a_{\mathrm{d}}=0.5\right)$. $\mathbf{d}$ optix-Gal4 expression in the dorsal and ventral subdomains of the optic lobe (green). e, f Changes in N activity (NRE-dVenus, green) upon DI RNAi under the control of optix-Gal4. DI (magenta) and Lsc (blue). The signal intensities of NRE-dVenus, DI, and Lsc within the white (control) and yellow boxes (DI RNAi) are plotted in the right panels. Asterisks indicate the optix-Gal4 positive areas outlined by white lines. e Partial D/ knockdown causes the fusion of the twin peaks of $\mathrm{N}$ activity (yellow box, arrows). f Strong DI knockdown causes the loss of DI expression and $\mathrm{N}$ activity (yellow box). $\mathbf{g} \mathrm{A}$ schema illustrating trans-activation and cis-inhibition at the wavefront. $\mathbf{h}$ Expression level of full-length $N$ (white) is reduced behind the wavefront (Lsc, blue). Signal intensities of $N$ (black) and Lsc (blue) within the white box are plotted in the right panel. Arrows indicate the reduction of $\mathrm{N}$ expression level. $\mathbf{i}, \mathbf{j}$ Expression level of full-length $\mathrm{N}$ (white) is upregulated in $\mathrm{Dl}$ mutant clones visualized by the absence of GFP (green in $\mathbf{i}$, blue in $\mathbf{j}$ ) behind the wavefront (Lsc, blue). i Signal intensities of $\mathrm{N}$ (black) within the dotted box are plotted in the right panel. Arrow indicates the upregulation of $\mathrm{N}$ expression level. $\mathbf{j} \mathrm{N}$ (magenta) is accumulated along the cell membrane (Ecad, green) in D/ mutant clones. The dotted box is magnified in the right panel. Scale bars indicate $20 \mu \mathrm{m}$.

cells adjacent to the proneural wavefront. Thus, $\mathrm{Dl}$ expression at the wavefront is responsible for generating the first and second $\mathrm{N}$ activity peaks.

The intuitive interpretation of the above results is that the Dl expression at the wavefront activates $\mathrm{N}$ signaling in the adjacent $\mathrm{NE}$ cells (first peak) and NBs (second peak). $\mathrm{N}$ activity is downregulated at the wavefront between the twin peaks of $\mathrm{N}$ activity (Fig. 2g). However, the first $\mathrm{N}$ activity peak partially overlaps with $\mathrm{Dl}$ expression in vivo (Fig. 1a-d). This is most likely because the proneural wave progresses in a medial-to-lateral orientation. While $\mathrm{N}$ activity establishes the twin peaks, the location of the Dl-expressing wavefront cells may change and partially overlap with the first $\mathrm{N}$ activity.

Delta triggers Notch degradation at the wavefront. The above results suggest that the strong $\mathrm{Dl}$ expression at the wavefront triggers quick inactivation of $\mathrm{N}$ signaling. Since the expression level of $\mathrm{N}$ is downregulated behind the wavefront, the expression of $\mathrm{Dl}$ may trigger $\mathrm{N}$ degradation (Fig. 2h). If this is the case, the expression level of $\mathrm{N}$ should be upregulated when the function of $\mathrm{Dl}$ is compromised. As expected, we observed striking upregulation of the full-length $\mathrm{N}$ protein, which accumulated along the plasma membrane visualized by DE-cadherin (Ecad), when $D l$ mutant clones were generated at the wavefront (Fig. $2 \mathrm{i}: n=15 / 25,2 \mathrm{j}: n=14 / 20$ ). In contrast, $N$ messenger RNA (mRNA) level was not affected in $D l$ mutant clones (Supplementary Figure 5). These results suggest that post-translational $\mathrm{N}$ degradation upon $\mathrm{Dl}$ expression is the basis of the nonlinear nature of cis-inhibition.

In our mathematical model, the expression level of full-length $\mathrm{N}$ protein is assumed to be constant, while the term $-k_{\mathrm{n}} N$ represents passive degradation of $\mathrm{N}$ signaling, which is independent of $\mathrm{Dl}$ expression. Considering the above experimental results, full-length $\mathrm{N}$ should be actively degraded through Dldependent cis-inhibition, which is incorporated into the model as active downregulation of $\mathrm{N}$ signaling in a Dl-dependent manner (Fig. 1e-g). Our model allows $N$ to be negative (Supplementary Figures 1 and 2), which might correspond to the presence of a repressor complex of $\mathrm{Su}(\mathrm{H})$ and Hairless $(\mathrm{H})^{8}$. We only use the parameter settings with which $N$ remains nonnegative in the following study.

Rab7 and Rab4 differentially colocalize with Delta. It has been reported that $\mathrm{Dl}$ and $\mathrm{N}$ expressed in the same cell form a complex ${ }^{14,34}$. Consistent observations are found between $\mathrm{N}$ and Serrate (Ser), the other transmembrane ligand of $\mathrm{N}^{35}$. The formation of the Dl-N complex in cis may be the cause of the rapid $\mathrm{N}$ degradation. It has been reported that protein degradation in late endosomes regulates $\mathrm{N}$ signaling ${ }^{15-24}$. However, these studies did not focus on the process of cis-inhibition. In order to examine if the degradation machinery in late endosomes is responsible for cis-inhibition at the proneural wavefront, we asked whether $\mathrm{N}$ and $\mathrm{Dl}$ are transported through the intracellular trafficking pathways.

Rab family small GTPases play diverse roles in intracellular trafficking. We systematically screened the Rab-EYFP library strains in which all of the endogenous Rab family genes were tagged with $\mathrm{EYFP}^{36}$. Since $\mathrm{N}$ is downregulated at the wavefront, we initially compared Dl expression with Rab-EYFP distribution. We found that Rab4 and Rab7 are colocalized with $\mathrm{Dl}$ at the wavefront outside the nucleus and inside the membranous Ecad signals (Fig. 3a-f). These puncta are most likely in the cytoplasm. We confirmed the results by using anti-Rab7 antibody and found that Dl, Rab4, and Rab7 colocalize in the same puncta (Fig. $3 \mathrm{~h})^{37}$. The proximity ligation assay (PLA) also suggested that Rab7 forms a complex with $\mathrm{Dl}$ in vivo (Fig. $3 \mathrm{~g}$ ).

Since Rab7 and Rab4 are known to play key roles in late and recycling endosomes, respectively ${ }^{38,39}$, we hypothesized that the Dl-N complex is transported to the Rab7-positive late endosomes and that $\mathrm{Dl}$ is recycled to the plasma membrane through the Rab4-positive recycling endosomes.

Interestingly, we found that Rab4 signals are found inside the Dl puncta, while Rab7 mainly accumulates on the surface of the Dl puncta at a higher magnification (Fig. 3i). Indeed, the colocalization index of Dl-Rab4 was greater compared with that of Dl-Rab7 (Fig. 3i, right panel), suggesting that Rab4-dependent $\mathrm{Dl}$ recycling is more dominant than Rab7-dependent Dl degradation. The less prominent colocalization of Dl with Rab7 may explain why $\mathrm{Dl}$ is not degraded in late endosomes.

Although $\mathrm{N}$ is downregulated at the wavefront, we occasionally observed minor colocalizations of $\mathrm{N}$ with Rab7 and Rab4 (Fig. 3j). The colocalization indices of $\mathrm{N}$ and Rab7/4 were significantly lower compared with those of $\mathrm{Dl}$ and Rab7/4 (Fig. 3k). Importantly, N colocalized with Rab7 more significantly compared with Rab4, suggesting that Rab7-dependent $\mathrm{N}$ degradation is more dominant than Rab4-dependent N recycling. Similarly, colocalization of DlGFP, which recapitulates $\mathrm{Dl}$ distribution pattern, and $\mathrm{N}$ was occasionally observed (Fig. 31 and Supplementary Figure 5c). These results support the hypothesis that $\mathrm{N}$ is mainly degraded in late endosomes at the wavefront in a Dl-dependent manner.

ESCRT complex downregulates Notch activity at the wavefront. When proteins are transported from early to late endosomes (or multivesicular bodies), Rab7 and the ESCRT complex (ESCRT-I-III) regulate protein degradation through the fusion of late endosomes with lysosomes ${ }^{21,38}$. Since Dl colocalizes with 

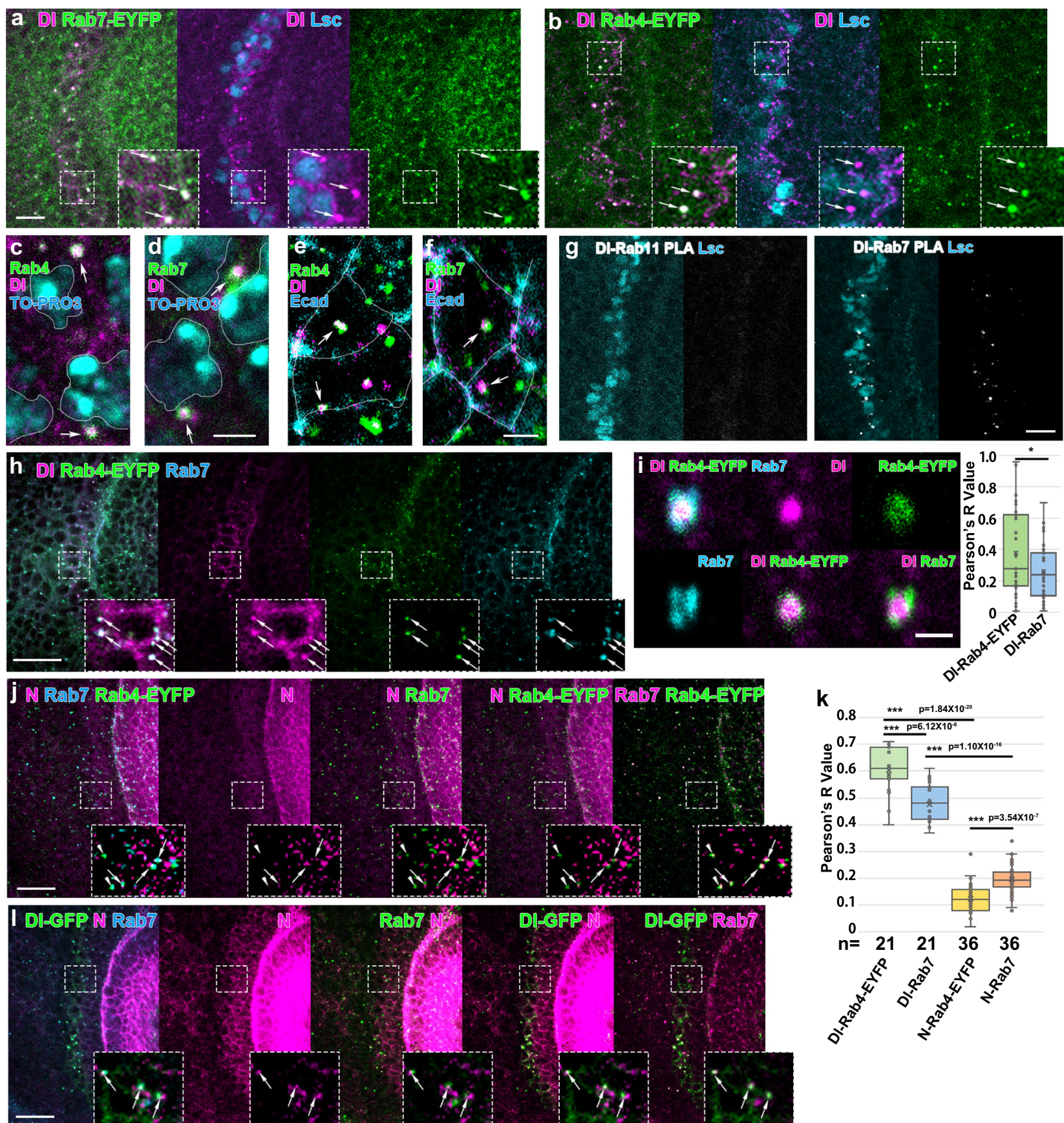

교

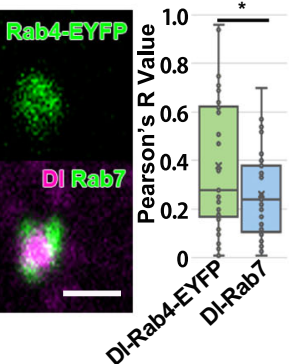



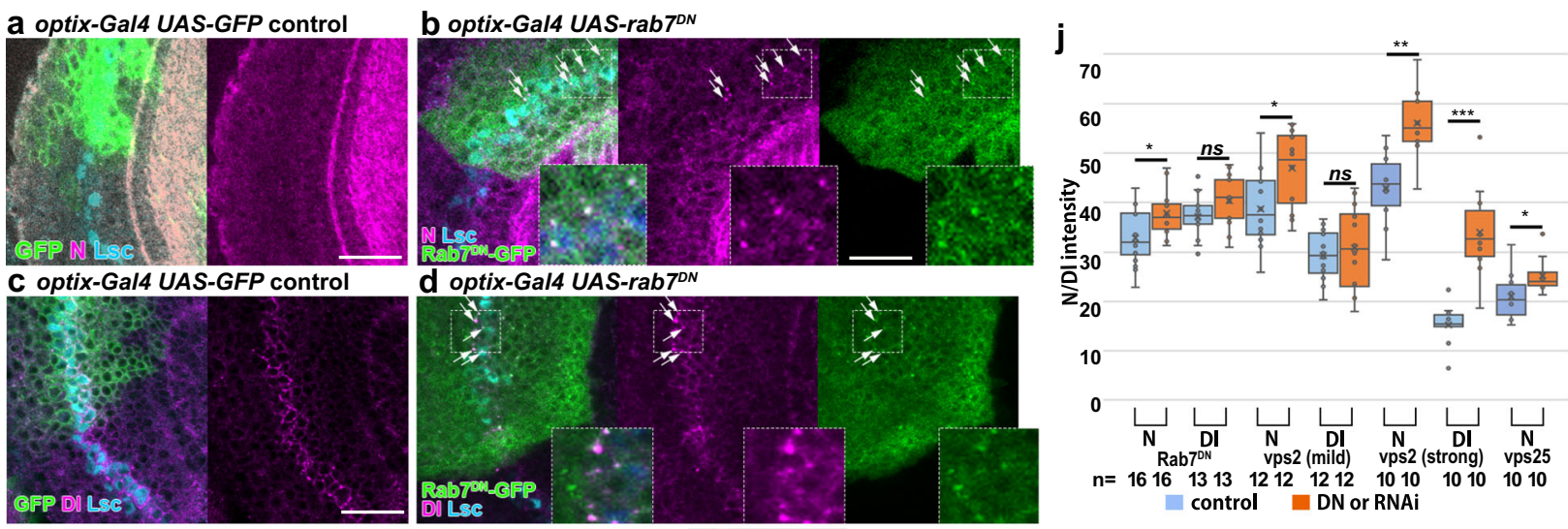

10

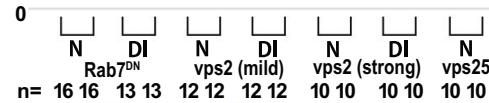

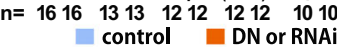
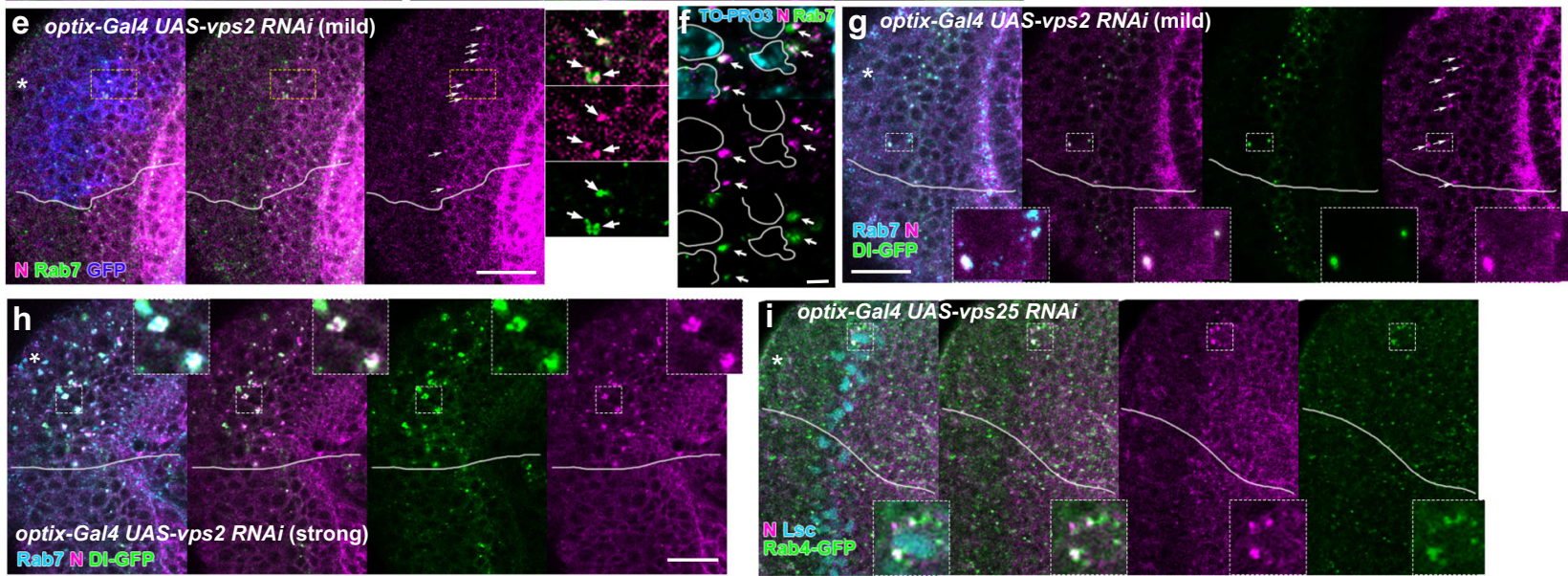

Fig. 4 Late endosomes are responsible for Notch degradation at the wavefront. a-d Expression of UAS-rab7DN-GFP under the control of optix-Gal4 (green) induces ectopic puncta of $\mathrm{N}$ (magenta in $\mathbf{a}, \mathbf{b}$ ) and DI (magenta in c, d) colocalized with Rab7DN-GFP (green, arrows) at the wavefront (Lsc, blue). The puncta of $\mathrm{N}$ and $\mathrm{DI}$ are not detected in the controls (a, $\mathbf{c}$ ). e-h vps2 RNAi under the control of optix-Gal4 induces ectopic puncta of N (magenta) at the wavefront (arrows) in the areas indicated by asterisks (marked by UAS-CD8GFP expression in $\mathbf{e}$ ). The N puncta are colocalized with Rab7 (green in $\mathbf{e}, \mathbf{f}$; blue in $\mathbf{g}, \mathbf{h}$ ) and DI-GFP (green in $\mathbf{g}, \mathbf{h}$ ). Mild and strong phenotypes are shown in (e-g) and (h), respectively. The $\mathrm{N}$ puncta are found outside the nucleus (TO-PRO3, blue). i vps25 RNAi under the control of optix-Gal4 induces ectopic puncta of N (magenta) that colocalize with Rab4-GFP (green) at the wavefront (arrows). The white dotted box area is enlarged in the right panels in (e, $\mathbf{g}-\mathbf{i})$. Scale bars indicate $20 \mu \mathrm{m}$ in (a-e, $\mathbf{g}-\mathbf{i})$ and $2 \mu \mathrm{m}$ in (f). j Quantification of the signal intensities of $\mathrm{N}$ and $\mathrm{DI}\left(p=0.008,0.108,0.180,0.613,0.0009,0.0001,0.042\left({ }^{\star} p<0.05,{ }^{\star \star} p<0.01,{ }^{\star \star \star} p<0.001\right.\right.$, n.s. not significant), two-sided $t$ test). The number of quantified areas is indicated. Cross, mean; center line, median; box limits, upper and lower quartiles; whiskers, $1.5 \times$ interquartile range.

when the function of the ESCRT protein is compromised in some experimental conditions ${ }^{20}$. Thus, late endosomes appear to be involved in multiple aspects of $\mathrm{N}$ signaling.

When we knocked down rab7 by expressing the dominantnegative form of $r a b 7$ or $r a b 7 \mathrm{RNAi}, \mathrm{N}$ protein level was slightly upregulated, showing punctate signals as visualized by $\mathrm{N}$ antibody (Figs. $4 \mathrm{a}, \mathrm{b}, \mathrm{j}$ and $5 \mathrm{a}, \mathrm{n}$ ). $\mathrm{N}$ and $\mathrm{Dl}$ colocalized with the Rab7 DN puncta (Fig. $4 \mathrm{a}-\mathrm{d}$ ). However, the $\mathrm{N}$ activity reporter was not significantly affected when $r a b 7$ was knocked down (see Fig. 5a).

Other late endosomal components may act redundantly with Rab7. We, therefore, focused on the functions of Vps family proteins that are involved in ESCRT complex function. When we knocked down $v p s 2$, a member of ESCRT-III, at the wavefront, $\mathrm{N}$ protein level was also upregulated, showing punctate signals (Fig. 4e, f). N and Dl colocalized in the Rab7- and Rab4-positive puncta (Fig. $4 \mathrm{e}-\mathrm{i}$ ).

We observed two distinct outcomes in $\mathrm{N}$ activity in the $v p s 2$ RNAi background. In $40 \%$ of the cases $(n=8 / 20)$, $\mathrm{N}$ activity was specifically upregulated in cells situated between the twin peaks nearby the wavefront indicated by Lsc expression. The level of full-length $\mathrm{N}$ protein was slightly upregulated forming a dotted pattern. At the same time, the twin peaks were fused to form a single peak (Figs. 4e, g, $\mathrm{j}$ and $5 \mathrm{~d}-\mathrm{f}, \mathrm{n}$ and Supplementary Figure 6a), which is similar to the results of the partial knockdown of $D l$ shown above (Fig. 2e and Supplementary Figure 4a). Since the upregulation of the level of full-length $\mathrm{N}$ were not as prominent as those found in $D l$ mutant clones (Fig. 2i, j), there might be as yet unknown mechanisms that regulate $\mathrm{N}$ degradation and cis-inhibition. Importantly, Dl expression level was not significantly affected (Fig. 4g, j and Supplementary Figure $6 \mathrm{~b}$ ), suggesting that $\mathrm{N}$ activation and the formation of the single peak are not caused by changes in $\mathrm{Dl}$ expression.

Since EGF signaling indirectly influences $\mathrm{N}$ activity $3,27,40$, the above phenotype may be the result of the changes in EGF activity. Since EGF activity as visualized by PntP1 staining was not significantly affected, the $\mathrm{N}$ activation phenotypes discussed above were not caused by indirect effects through EGF signaling (Supplementary Figure 7).

In the other $60 \%$ of the cases, $\mathrm{Dl}$ and full-length $\mathrm{N}$ proteins were widely upregulated (Figs. $4 \mathrm{~h}, \mathrm{j}$ and $5 \mathrm{~m}$ and Supplementary Figure $6 \mathrm{c}, \mathrm{d} ; n=12 / 20)$. Low-level $\mathrm{N}$ activity was observed in a wide area encompassing the wavefront (Fig. $5 \mathrm{~m}$ and Supplementary Figure 6c), which may be related to the hyper-activation of $\mathrm{N}$ 

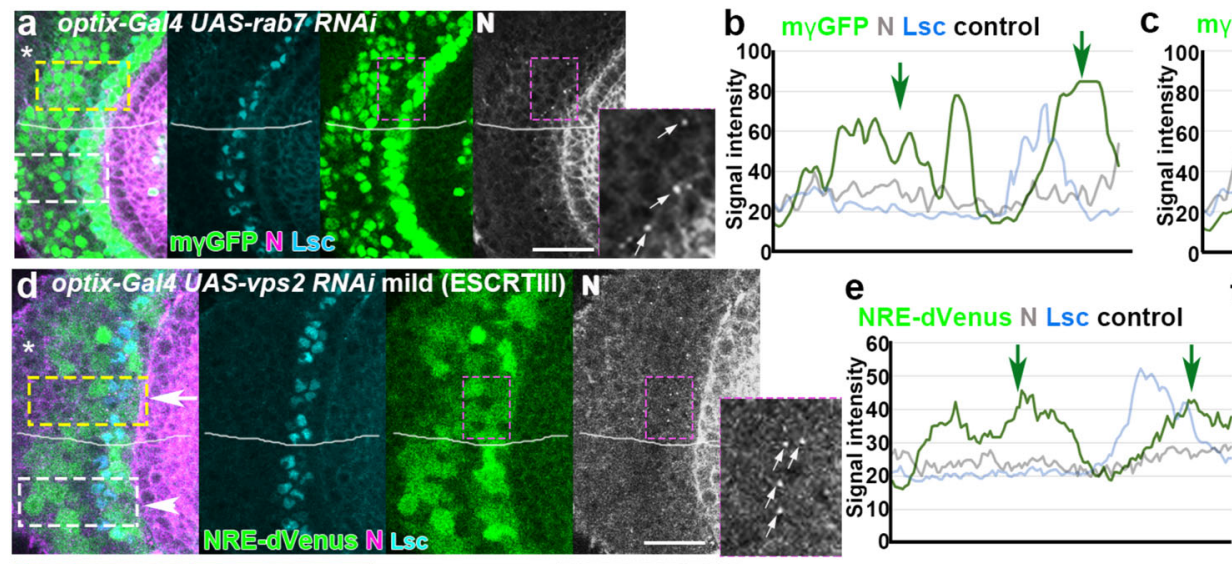

C myGFP N Lsc rab7 RNAi mild
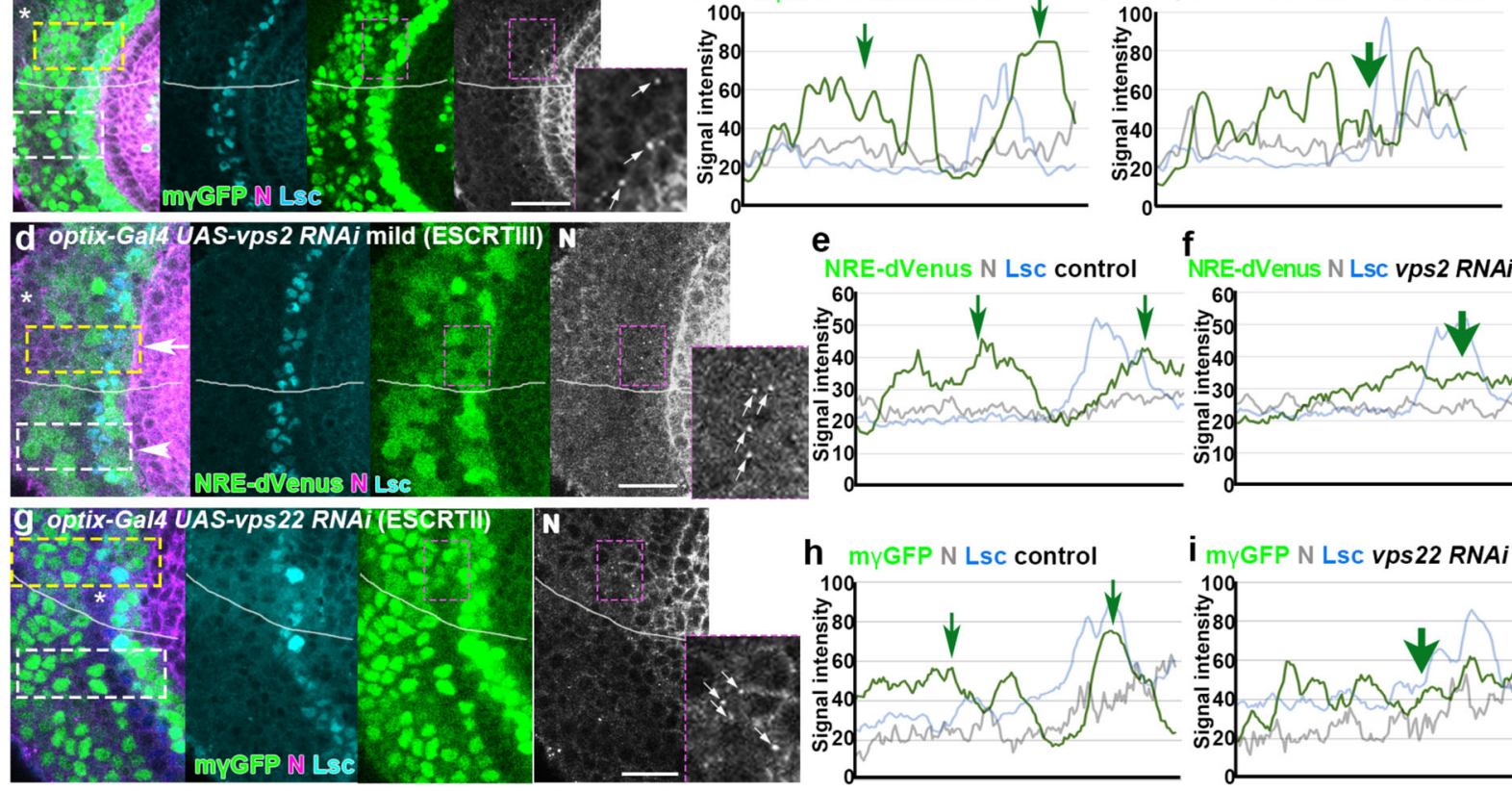

$f$ NRE-dVenus N Lsc vps2 RNAi mild
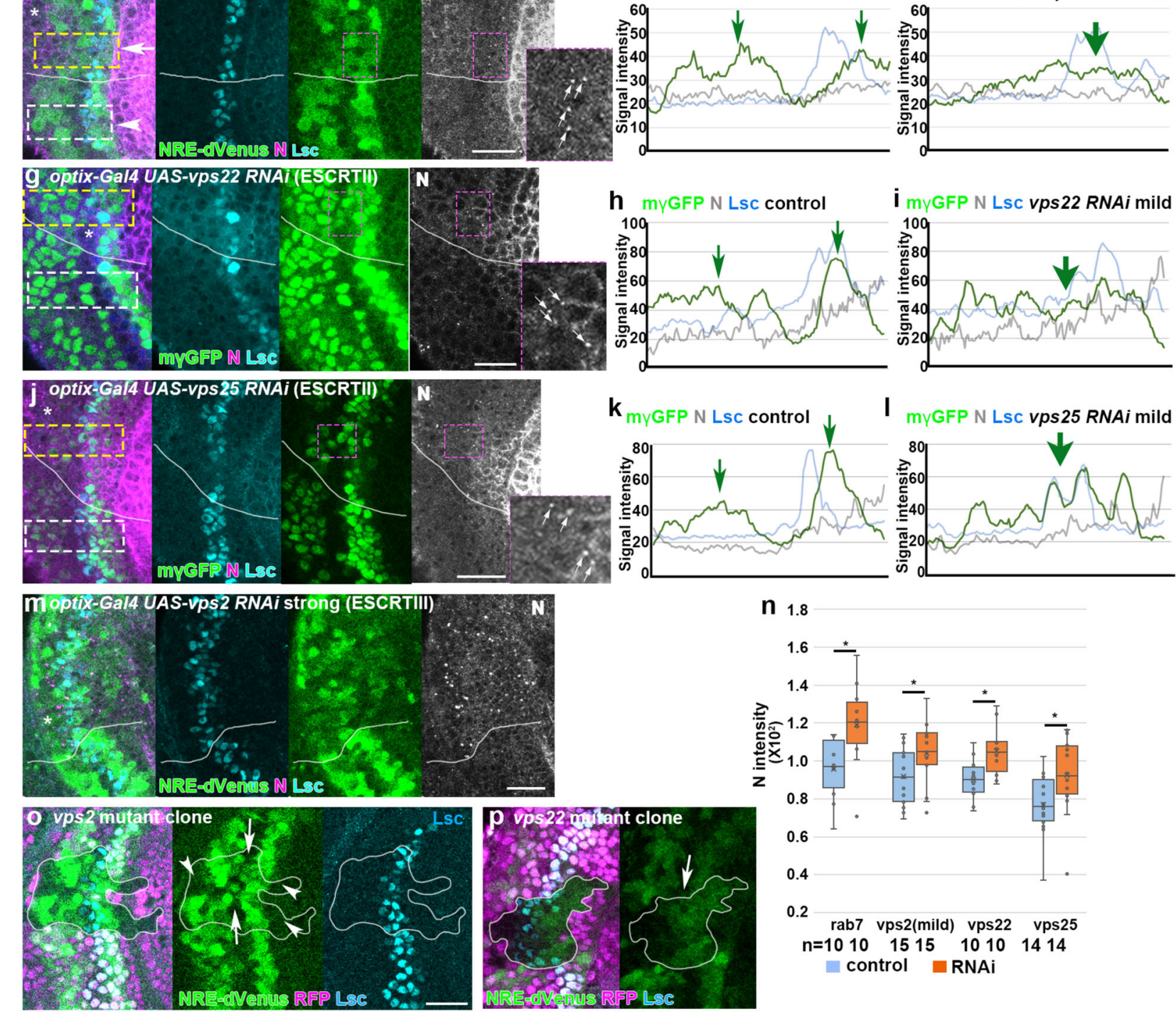

Fig. 5 Late endosomes are responsible for Notch inactivation at the wavefront. a-I RNAi knockdown of rab7 (a), vps2 (d), vps22 (g), and vps 25 (j) under the control of optix-Gal4 induces ectopic puncta of N (magenta or white, arrows) and fusion of the twin activation peaks of N (m $\gamma$ GFP in $\mathbf{a}, \mathbf{g}$, $\mathbf{j}$ and NRE$\mathrm{dVenus}$ in $\mathbf{d}$, green) at the wavefront (Lsc, blue). The magenta dotted boxes are magnified in the right panels showing the ectopic puncta of $\mathrm{N}$. Asterisks indicate the optix-Gal4 positive areas outlined by white lines. The signal intensities of $\mathrm{N}$ activity (green), $\mathrm{N}$ expression (gray), and Lsc (blue) within the white and yellow dotted boxes in ( $\mathbf{a}, \mathbf{d}, \mathbf{g}, \mathbf{j})$ are plotted in $(\mathbf{b}, \mathbf{e}, \mathbf{h}, \mathbf{k}$; controls) and ( $\mathbf{c}, \mathbf{f}, \mathbf{i}, \mathbf{l} ;$ RNAi), respectively. Green arrows indicate the $N$ activity peaks. $\mathbf{m}$ vps2 RNAi with a strong phenotype induces ectopic $\mathrm{N}$ puncta (magenta and white) and uniform $\mathrm{N}$ activity (NRE-dVenus, green) in a wide area (asterisk). Lsc (blue). $\mathbf{n}$ Quantification of N intensity ( $p=0.020,0.047,0.017,0.038\left({ }^{*} p<0.05\right)$, two-sided $t$ test). Numbers of quantified areas are indicated. Cross, mean; center line, median; box limits, upper and lower quartiles; whiskers, $1.5 \times$ interquartile range. $\mathbf{0}$, $\mathbf{p}$ Fusion of the twin peaks of $\mathrm{N}$ activity (NRE-dVenus, green) in vps2 and vps22 mutant clones, respectively, visualized by the absence of RFP (magenta). Arrows indicate the upregulation of $\mathrm{N}$ activity between the twin peaks. Arrowheads indicate the absence of $\mathrm{N}$ activation in cells adjacent to the wavefront (Lsc, blue). Scale bars indicate $20 \mu \mathrm{m}$.

signaling and/or suppression of $\mathrm{N}$ activity in $v p s 2$ mutant cells ${ }^{15-18,20}$. Since these phenotypes accompany smaller brain sizes compared with the brains showing the specific $\mathrm{N}$ activation phenotype discussed above, we assume that the mild RNAi effect caused the specific $\mathrm{N}$ activation between the twin peaks (Fig. 5d).
We repeated the same experiments for the other ESCRT complex genes and found essentially the same results (Fig. 5g-n and Supplementary Figure 8). Importantly, the specific N activation at the wavefront and the fusion of the twin peaks were reproducible in multiple RNAi conditions and mutant 
clones of $v p s 2$ and $v p s 22$ (Fig. 5g-p and Supplementary Figure 8). Note that the twin peaks were partially fused, while ectopic $\mathrm{N}$ activation was not found in cells apart from the wavefront in the vps2 and vps22 mutant clones (Fig. 5o, p; $n=9 / 15$ and 11/16). These results cannot be explained by the previously proposed roles of late endosomes that nonspecifically and uniformly degrade $\mathrm{N}$ and $\mathrm{Dl}$. Thus, late endosomes play specific roles in $\mathrm{N}$ degradation that are essential for cis-inhibition at the proneural wavefront in addition to the roles in uniform nonspecific $\mathrm{N}$ degradation.

Delta transport from late endosomes to recycling endosomes. We hypothesize that the Dl-N complex is transported to late endosomes, where only $\mathrm{N}$ is degraded (Fig. 6o). We also hypothesize that $\mathrm{Dl}$ is released from late endosomes prior to its degradation and is recycled to the plasma membrane through recycling endosomes, because Rab4 colocalized with Dl more significantly compared with Rab7 (Fig. 3i).

When we knocked down rab4 with RNAi, Dl expression was accumulated at the wavefront in a milder condition at $25^{\circ} \mathrm{C}$ (Fig. 6a, b, m) and the colocalization of $\mathrm{Dl}$ with $\mathrm{Rab} 7$ was significantly increased (Fig. 6b). Interestingly, Dl expression was downregulated in a stronger RNAi condition at $30^{\circ} \mathrm{C}$ (Fig. $6 \mathrm{c}, \mathrm{m}$ ). These results suggest that $\mathrm{Dl}$ is retained in Rab7-positive late endosomes and is degraded together with $\mathrm{N}$ when the function of recycling endosomes is eliminated. Furthermore, overexpression of rab4 ${ }^{D N}$ mimicked the effects of rab4 RNAi in the milder condition, and aggregated distribution of $\mathrm{Dl}$ colocalized with Rab7 in the cytoplasm of cells at the wavefront (Supplementary Figure 9). Similar results were demonstrated in cells mutant for the components of recycling endosomes in the central brain ${ }^{41}$.

It is known that late endosomes become acidic in the course of fusion with lysosomes, by which proteins are degraded ${ }^{42}$. The decrease in $\mathrm{pH}$ may trigger $\mathrm{Dl}$ dissociation from the $\mathrm{Dl}-\mathrm{N}$ complex. Rabconnectin (Rbcn) is a family of proteins that regulate endocytic trafficking by regulating the assembly and activity of vacuolar-ATPase (V-ATPase), which is responsible for the acidification of intracellular compartments ${ }^{43,44}$. Thus, we focused on the roles of $R b c n$ and $v h a 68-2$, a member of V-ATPase genes, in this process. In vha68-2 mutant clones, Dl was aggregated and colocalized with Rab7 (Fig. 6d, e). When vha682 was knocked down, signals indicating acidic cellular components were significantly reduced as visualized by Lysotracker (Supplementary Figure 10c). At the same time, $\mathrm{N}$ degradation was blocked, and the level of $\mathrm{N}$ protein was upregulated at the wavefront (Fig. $6 \mathrm{f}-\mathrm{j}$ and Supplementary Figure 10). In this condition, we observed ectopic colocalization of $\mathrm{Dl}$ and $\mathrm{N}$ in $\mathrm{Rab} 7$ and Rab4-positive puncta (Fig. $6 \mathrm{~h}-\mathrm{j}$ ), suggesting that acidification of the endocytic pathway triggers $\mathrm{Dl}$ dissociation from the $\mathrm{Dl}-\mathrm{N}$ complex and $\mathrm{N}$ degradation. These results are consistent with the impaired endosomal acidification and accumulation of $\mathrm{N}$ in late endosomes and lysosomes in vha68-2 mutant clones in eye imaginal disc ${ }^{45}$.

Based on the above results, we hypothesize that the Dl-N complex in Rab7-positive late endosomes is dissociated upon $\mathrm{pH}$ decrease. As a result, $\mathrm{N}$ is degraded in lysosomes, while $\mathrm{Dl}$ is recycled to the cell membrane through Rab4-positive recycling endosomes. Since $\mathrm{pH}$ increase upon vha68-2 RNAi caused Dl colocalization with Rab7 and Rab4 (Fig. 6h, i), Dl-N complex may be localized to either late endosomes or recycling endosomes when acidification is compromised.

If $\mathrm{Dl}$ is transported from late to recycling endosomes, Dl should more strongly colocalize with Rab7 when rab4 is knocked down together with vha68-2. To test this possibility, we compared colocalization of Dl with Rab7 in vha68-2 RNAi and vha68-2 rab4 double RNAi backgrounds. Indeed, the colocalization of $\mathrm{Dl}$ with Rab7 was significantly increased by knocking down rab4 together with vha68-2 (Fig. $6 \mathrm{~h}, \mathrm{k}, \mathrm{l}, \mathrm{n}$ ). These results support the model shown in Fig. 60.

The second Notch activity controls neurogenesis. The first peak of $\mathrm{N}$ activity is responsible for regulating the speed of proneural wave propagation 3,27 . What is the biological function of the second peak? We carefully compared the pattern of $\mathrm{N}$ activity and genes that are specifically expressed in the NBs behind the wavefront. Interestingly, the expression of one of the temporal transcription factors, Klu, coincides with the second peak of $\mathrm{N}$ activity (Fig. 7a, 1) ${ }^{31,46}$.

So far, Hth, Klu, Ey, Slp, D, and Tll show sequential expression in the medulla $\mathrm{NBs}^{30,31}$. However, the mutual interactions between $\mathrm{Hth}, \mathrm{Klu}$, and Ey have not been identified thus far. Since Hth is upregulated in NEs prior to the arrival of the proneural wave and is continuously expressed in NEs and NBs (Fig. 7l), the mechanism that regulates Klu expression remains unclear. The $\mathrm{N}$ activity in the second peak may regulate the onset of Klu expression in NBs.

We initially examined the effect of the complete elimination of Dl function by generating $D l$-null mutant clones in which the proneural wave is accelerated ${ }^{27}$. Since Klu expression persists for a long time following its induction at the second $\mathrm{N}$ activity peak, the premature NB differentiation would still show a persistent Klu expression behind the accelerated wavefront. However, Klu expression was eliminated in $\mathrm{Dl}$ mutant cells except for the cells situated along the boundary between the Dl-positive cells (Fig. 7b, $n=16$ ). The residual Klu expression along the clone boundary may be due to the non-autonomous effect of $\mathrm{N}$ trans-activation. In contrast, the expression Hth, Ey, and Slp was not significantly affected in $D l$ mutant clones (Supplementary Figure 11a-c). These results suggest that $\mathrm{N}$ signaling is indeed necessary for Klu expression in NBs.

Since we do not have a technique that specifically inactivates the second $\mathrm{N}$ peak, we made use of the partial knockdown of $D l$ and $v p s 2$, resulting in the loss of the gap between the twin peaks and, consequently, the fusion of the twin peaks. Interestingly, Klu was precociously expressed in the newborn NBs under these conditions without significantly affecting the proneural wave progression (Fig. $7 \mathrm{c}, \mathrm{d}$ ), suggesting that the second $\mathrm{N}$ activity peak in NBs indeed triggers the expression of Klu. Note that Klu expression is not activated in the first peak of $\mathrm{N}$ activity in NEs (Fig. 7a, l). Klu expression may require additional genetic factors that are specific to NBs.

We previously demonstrated that $\mathrm{Hth}$ expression in the newborn NBs promotes the production of brain-specific homeobox (Bsh)-positive neurons that form the innermost concentric

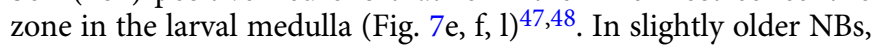
Klu expression triggers the production of Runt (Run)-positive neurons, forming a concentric zone just outside the Bsh-positive neurons ${ }^{31}$. Consistent with the precocious Klu expression in NBs, we occasionally observed Run-positive neurons in an area inside the Bsh-positive neurons in $D l$ and $v p s 2$ RNAi conditions (Fig. $7 \mathrm{~g}$, $h$, i). These defects were restricted to the dorsal part of the brain, most likely due to the stronger expression of optix-Gal4 in the dorsal brain (Fig. 7e). The Klu expression in the newborn NBs, which only express $\mathrm{Hth}$ in the control background, might cause the production of Run-positive neurons earlier than Bsh-positive neurons, resulting in their abnormal distributions in the medulla (Fig. 7l). Note that Hth expression is widely found in NE and NB cells, and is not affected by $\mathrm{Klu}^{31}$. Therefore, the expression of Hth and production of Bsh-positive neurons should not be affected. 

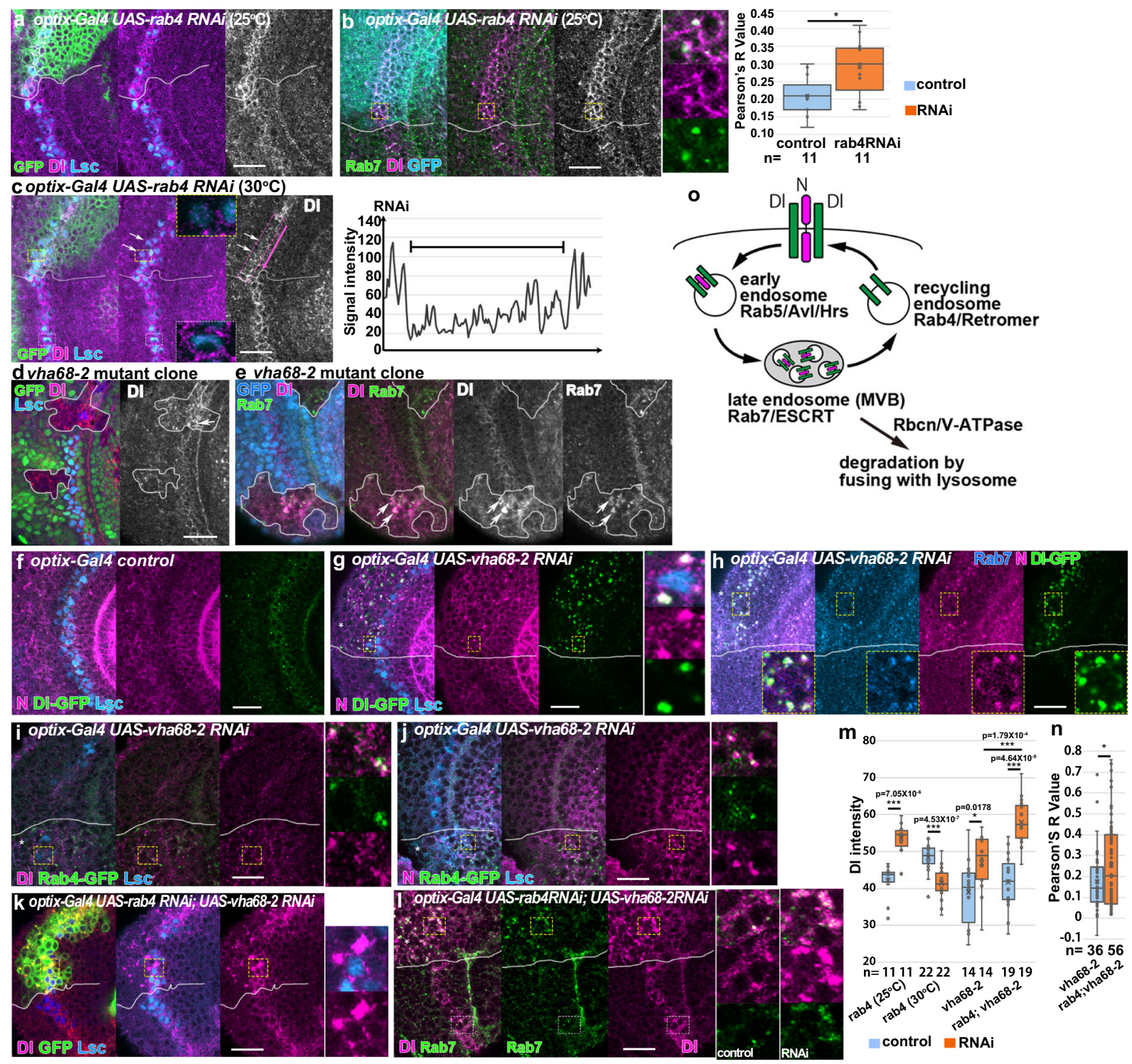

Fig. 6 Delta transport from late endosomes to recycling endosomes. a, b DI aggregation (magenta or white) upon rab4 RNAi (optix-Gal4, green in a, blue in b) at $25^{\circ} \mathrm{C}$. Lsc, blue (a) and Rab7, green (b). DI colocalizes with Rab7 (b). The white dotted box is magnified. The colocalization of DI with Rab7 is quantified ( $p=0.016$ ( ${ }^{*} p<0.05$ ), two-sided $t$ test). c Loss of Dl expression (arrows) by rab4 RNAi (optix-Gal4, green) at $30^{\circ} \mathrm{C}$ at the wavefront (Lsc, blue). The white dotted boxes are enlarged showing the decrease of $\mathrm{DI}$ in the mutant area (yellow) compared with the control area (white). Signals in the magenta dotted box are plotted in the right panel. The magenta arrow indicates the orientation along the $x$-axis. d, e DI is upregulated in vha68-2 mutant clones (arrows) visualized by the absence of GFP (green in d, blue in e) at the wavefront (Lsc, blue in a). Ectopic DI is accumulated in Rab7-positive structures (green in e). f Control distributions of N (magenta), DI-GFP (green), and Lsc (blue). g-j vha68-2 RNAi under the control of optix-Gal4. Distribution of ectopic N puncta (magenta in $\mathbf{g}-\mathbf{j}$ ), DI-GFP (green in $\mathbf{g}, \mathbf{h}$ ), DI (magenta in $\mathbf{i}$ ), Rab7 (blue in $\mathbf{h}$ ), and Rab4-GFP (green in $\mathbf{i}, \mathbf{j}$ ) is compared behind the wavefront (Lsc, blue in $\mathbf{g}, \mathbf{i}, \mathbf{j}$ ). $\mathbf{k}$, I rab4 vha68-2 double RNAi under the control of optix-Gal4 (GFP, green in $\mathbf{k}$ ). DI (magenta), Rab7 (green in I), and Lsc (blue in $\mathbf{k}$ ). The yellow and white dotted boxes in RNAi and control areas, respectively, are magnified in the right panels. Asterisks indicate the optix-Gal4-positive areas outlined by white lines. $\mathbf{m}$ DI intensity quantification in different RNAi conditions ( $p$ values are indicated inside the panel, ${ }^{*} p<$ $0.05,{ }^{\star \star \star} p<0.001$, two-sided $t$ test). $\mathbf{n}$ Quantification of the colocalization between DI and Rab7 ( $p=0.041$ ( ${ }^{\star} p<0.05$ ), two-sided $t$ test). b, m, $\mathbf{n}$ Numbers of quantified areas are indicated. Cross, mean; center line, median; box limits, upper and lower quartiles; whiskers, $1.5 \times$ interquartile range in the box plots. - Putative intracellular mechanisms that degrade $\mathrm{N}$ and recycle DI. Scale bars, $20 \mu \mathrm{m}$.

We have demonstrated that Bsh-positive neurons give rise to Mil medulla neurons ${ }^{47,48}$. Similarly, the results of clonal analysis using run-Gal4 demonstrate that Run-positive neurons differentiate into Mi4 and TmY16 neurons (Fig. 7j, k; $n=60$ and 49 , respectively). The neuronal-type TmY16 has not been documented based on its projection pattern in the medulla, lobula, and lobula plate (Dr. Kazunori Shinomiya, personal communication). Thus, the temporal regulation of the $\mathrm{N}$ dynamics at the proneural wavefront controls the temporal pattern of neuronal-type specification through the expression of the temporal transcription factor Klu.

\section{Discussion}

In this study, we incorporated nonlinearity in cis-inhibition to the mathematical model of the proneural wave and reproduced the twin activation peaks of $\mathrm{N}$ signaling at the wavefront as observed in vivo. The fast nonlinear dynamics of cis-inhibition compared 

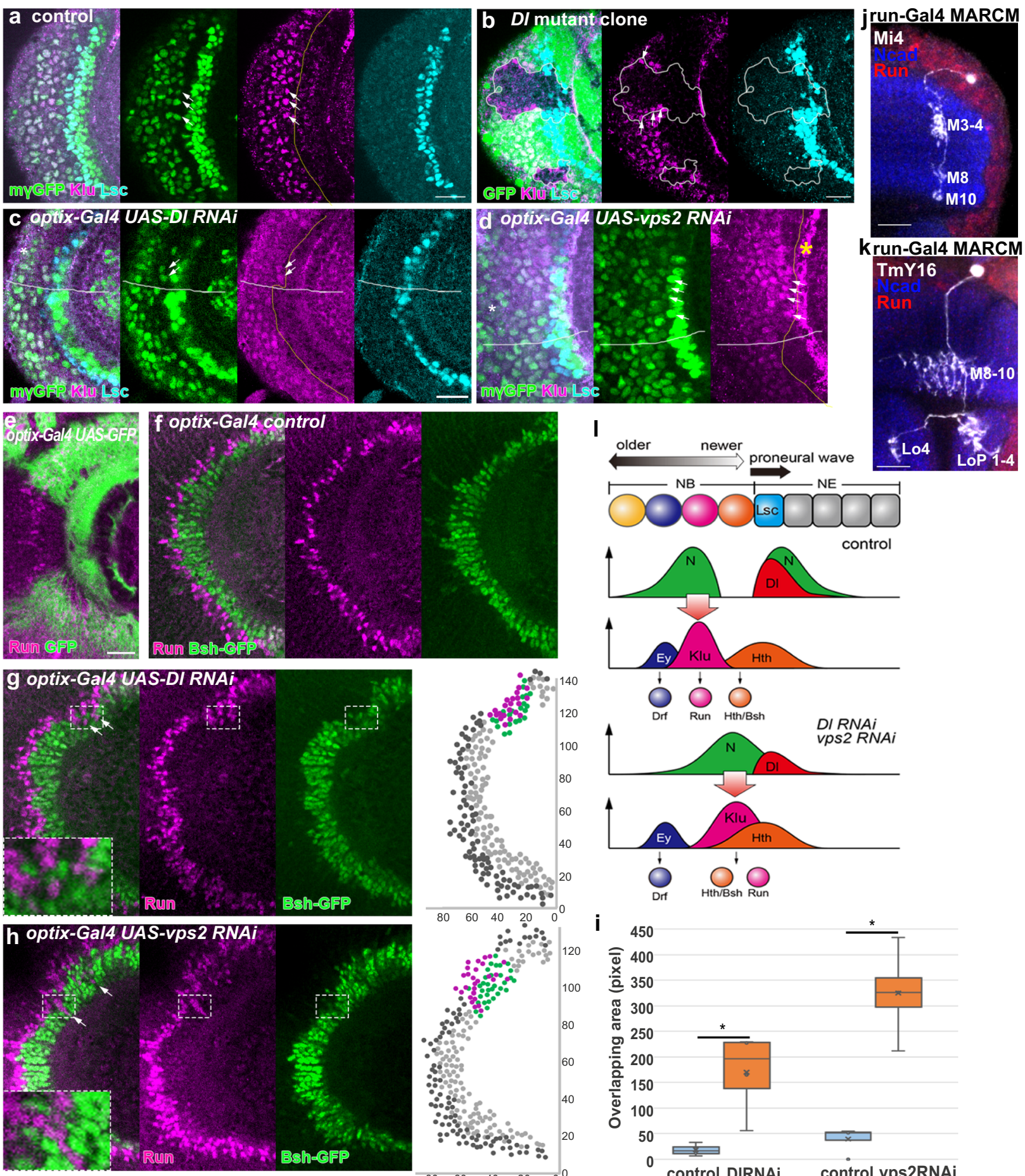

krun-Gal4 MARCM
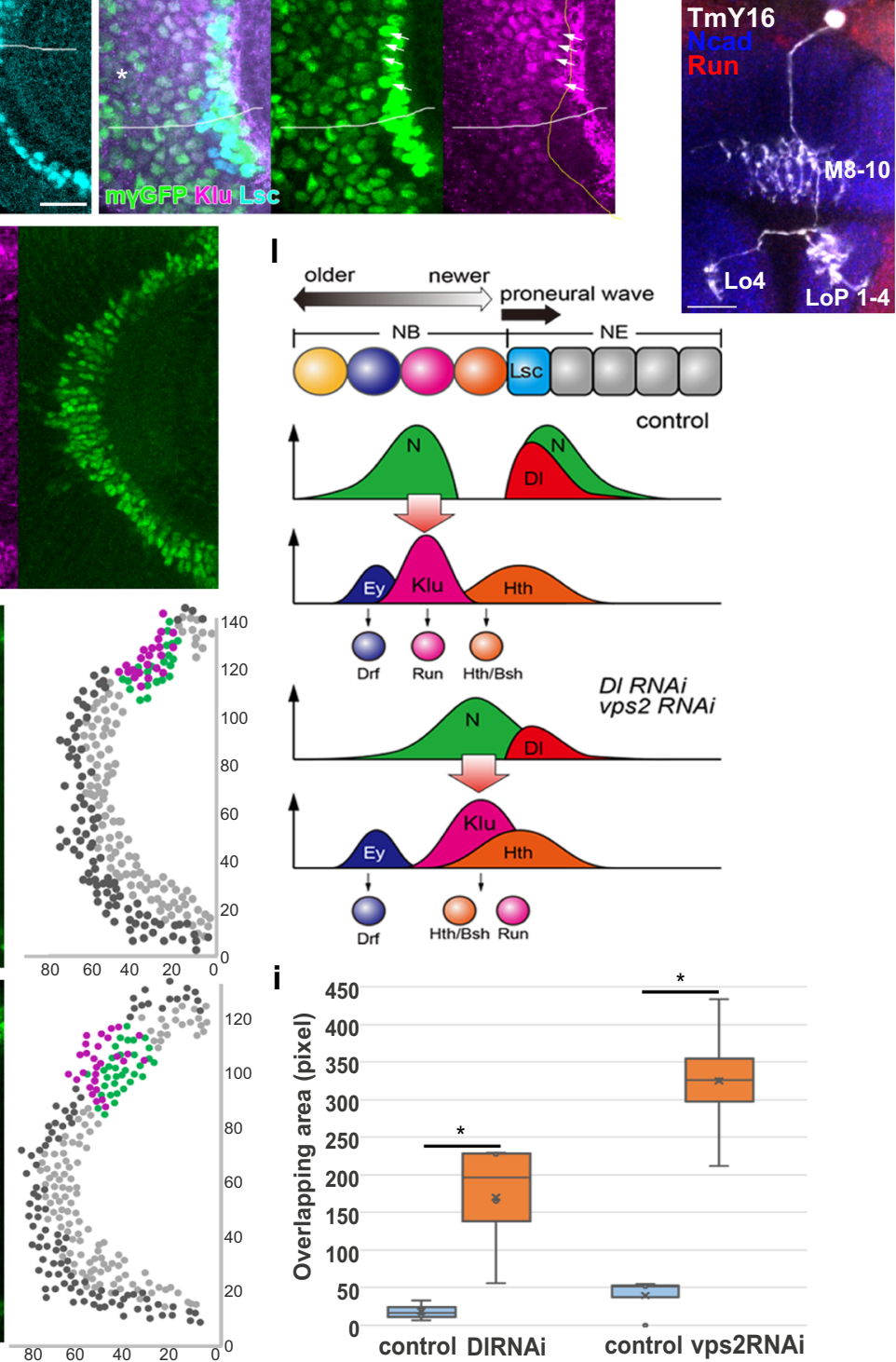

with those of the gradual kinetics of trans-activation is consistent with the results of the in vitro cell culture system ${ }^{11}$. The differential kinetics of trans-activation and cis-inhibition provide the rich dynamics of $\mathrm{N}$ activity that enable the formation of the twin activation peaks and the regulation of the temporal patterning of neurogenesis.

According to the previous literatures, the upregulation of $\mathrm{Dl}$ expression may induce the clustering of $\mathrm{N}$ and $\mathrm{Dl}^{14,34,35}$, which leads to an acute suppression of $\mathrm{N}$ signal activity via cis-inhibition (Fig. 6o). Consistent with this idea, the partial knockdown of $D l$ caused an upregulation of $\mathrm{N}$ activity between the twin peaks and their fusion, which reflects the failure in cis-inhibition. The expression level of full-length $\mathrm{N}$ is temporally downregulated behind the wavefront, which is derepressed in $D l$ mutant clones (Fig. 2h, i), suggesting that cis-inhibition is triggered by $\mathrm{N}$ degradation in response to $\mathrm{Dl}$ expression.

We essentially focused on the roles of intracellular trafficking in regulating $\mathrm{N}$ degradation and cis-inhibition (Fig. 6o). RNAi and mutant clones of ESCRT complex genes caused the fusion of the twin peaks of $\mathrm{N}$ activity, which is similar to the result of the partial $D l$ knockdown. We assume that the ESCRT complex regulates $\mathrm{N}$ degradation in the presence of high levels of $\mathrm{Dl}$ 
Fig. 7 The second Notch peak regulates the temporal patterning of neurogenesis. a Klu expression (magenta) coincides with the second peak of $\mathrm{N}$ activity (myGFP, green) as indicated by arrows behind the wavefront (Lsc, blue). b Klu expression (magenta) is eliminated in DI mutant clones visualized by the absence of GFP (green), except for cells at the clone boundary (arrows). Lsc visualizes the accelerated wavefront inside the DI mutant clones (blue). c, $\mathbf{d}$ Fusion of the twin peaks of $\mathrm{N}$ activity (myGFP, green) and precocious Klu expression (magenta) indicated by arrows upon partial knockdown of $\mathrm{DI}$ (c) and vps2 (d). Asterisks indicate the optix-Gal4-positive areas outlined by white lines. Yellow lines outline Klu-expressing NBs. The wavefronts are not accelerated (Lsc, blue). e optix-Gal4 is expressed in the dorsal (top) and ventral (bottom) subdomains of the optic lobe (GFP, green). GFP signals are stronger in the dorsal subdomain. Run (magenta). $\mathbf{f}$ Cells expressing Bsh-GFP (green) and Run (magenta) form the concentric zones that are complementary with each other. $\mathbf{g}$, h Ectopic Run-positive cells (magenta) are found within the concentric zone expressing Bsh-GFP (green) upon partial knockdown of $\mathrm{Dl}$ and vps2 as indicated by arrows. Positions of Run and Bsh-positive cells are plotted in magenta and green within the defective areas and in black and gray in the other areas, respectively, in the right panels. i Overlaps between Run- and Bsh-positive areas are quantified in DI and vps RNAi backgrounds ( $p=0.034,0.009$ ( ${ }^{\star} p<0.05,{ }^{\star \star} p<0.01$ ), two-sided $t$ test, $n=4$, number of brain samples). Cross, mean; center line, median; box limits, upper and lower quartiles; whiskers, $1.5 \times$ interquartile range. j, k Morphologies of Run-positive medulla neurons, Mi4 (j) and TmY16 (k) visualized by runGal4 UAS-CD8GFP MARCM clones (white). Run (red) and Ncad (blue). Layers in the medulla (M), lobula (Lo), and lobula plate (Lop). I Schematics of the temporal patterning of neurogenesis through $\mathrm{N}$ activity and Klu expression. Hth- and Klu-positive NBs produce Bsh- and Run-positive neurons, respectively. Fusion of the twin peaks of Notch activity leads to precocious Klu expression, which precociously produces Run-positive neurons. Scale bars, $20 \mu \mathrm{m}$.

expression. Since the expression level of full-length $\mathrm{N}$ was only partially upregulated, forming a dotted pattern by knocking down rab7 and ESCRT genes, there might be as yet unknown mechanisms that regulate $\mathrm{N}$ degradation (Fig. 5, Supplementary Figure 6).

We assumed that the clustering of $\mathrm{N}$ and $\mathrm{Dl}$ is the trigger of $\mathrm{N}$ degradation. However, Dl is not degraded at the wavefront, as evident from its strong membrane localization, despite its localization in Rab7-positive late endosomes. Since the colocalization of $\mathrm{Dl}$ with Rab4 is more prominent than that with Rab7, recycling of $\mathrm{Dl}$ to the plasma membrane may be dominant compared with its degradation (Fig. 3i). Indeed, a mild knockdown of rab4 caused Dl accumulation in Rab7-positive late endosomes, while its severe knockdown induced Dl degradation (Fig. 6a-c). Colocalization of $\mathrm{Dl}$ with $\mathrm{Rab} 7$ upon blocking acidification in late endosomes was further enhanced by the simultaneous knockdown of vha68-2 and rab4 (Fig. 61, n). These results suggest that $\mathrm{Dl}$ is dissociated from the $\mathrm{Dl}-\mathrm{N}$ complex in late endosomes, and is recycled to the plasma membrane through recycling endosomes (Fig. 6o).

The mathematical models in Fig. 1 do not explicitly consider the degradation of $\mathrm{N}$ protein upon its cis-interaction with $\mathrm{Dl}$. We further improved the model by considering full-length $\mathrm{N}(F)$ and active form of $\mathrm{N}(S)$. In a wide range of parameter settings, this model reproduces the formation of the twin peaks of $\mathrm{N}$ activity, fast degradation, and gradual recovery of the expression level of full-length N (Supplementary Figure 12). Although Dl function is thought to be inhibited when $\mathrm{Dl}$ and $\mathrm{N}$ interact in cis $^{11}$, we did not include this reaction in the model, because we do not have any observation that suggests cis-inhibition of $\mathrm{Dl}$ in the course of the proneural wave progression.

Furthermore, we demonstrated a role of $\mathrm{N}$ signaling in regulating the temporal patterning of neural progenitor cells (NPCs) by focusing on the transition of the temporal transcription factor $\mathrm{Klu}$, whose expression is upregulated in the medulla $\mathrm{NBs}^{31}$. We showed that $\mathrm{Dl}$ remotely regulates Klu expression behind the proneural wave through the second peak of $\mathrm{N}$ activity. Thus, fine temporal regulation of $\mathrm{N}$ activity through cis-inhibition plays essential roles in the temporal patterning of neurogenesis in the fly brain.

In the NPCs of the developing cerebral cortex, the temporal dynamics of $\mathrm{N}$ activity also play important roles in the temporal patterning of neurogenesis and gliogenesis ${ }^{49}$. In this process, the basic helix-loop-helix transcription factors show oscillatory expression in NPCs and N signaling appears to perform lateral inhibitory feedback during NPC differentiation. The roles of cisinhibition in this process remain largely elusive. It will be interesting to see how the molecular mechanisms revealed in the current study are conserved in a wide variety of developmental processes.

\section{Methods}

Mathematical modeling and numerical simulation. The differential equations were calculated using the explicit finite difference method with the zero flux boundary condition as described previously ${ }^{3}$. The mesh size of the square grid model is equal to the cell size ( $\mathrm{d} x=2,25 \times 25$ cells), and the time step size is 0.01 .

Four-variable model: The model contains four equations and four variables. $E$ is a composite variable for EGF ligand concentration and EGF signaling. $N$ represents $\mathrm{N}$ signal activity. $D$ and $A$ represent expression levels of $\mathrm{Dl}$ and AS-C, respectively. $d_{\mathrm{e}}$ is the diffusion coefficient of EGF. $d_{\mathrm{t}}$ and $d_{\mathrm{c}}$ represent magnitudes of the transactivation and cis-inhibition, respectively. $i, l$ and $j, m$ are integers indicating the location of a cell along the $x$ and $y$ axes, respectively. $l$ and $m$ indicate the location of four cells adjacent to a cell indicated by $i$ and $j$. The initial conditions for $A, E$, and $D$ in the anterior-most cells $(x=1,2,3)$ are $A=0.90,0.31$, and $0.02 ; E=$ $0.054,0.021$, and 0.0016 ; and $D=0.062,0.021$, and 0.0013 , respectively, to stabilize the proneural wave progression. The initial condition of $N$ is 0 in all cells.

$$
\begin{array}{ll}
\frac{\mathrm{d} E_{i, j}}{\mathrm{~d} t}=d_{\mathrm{e}} \Delta E_{i, j}-k_{\mathrm{e}} E_{i, j}+a_{\mathrm{e}} A_{i, j}\left(1-A_{i, j}\right) & (1-1) \\
\frac{\mathrm{d} N_{i, j}}{\mathrm{~d} t}=-k_{\mathrm{n}} N_{i, j}+d_{\mathrm{t}} \sum_{l, m \in \Lambda_{i, j}} D_{l, m}-d_{\mathrm{c}} D_{i, j} & (1-2) \\
\frac{\mathrm{d} D_{i, j}}{\mathrm{~d} t}=-k_{\mathrm{d}} D_{i, j}+a_{\mathrm{d}} A_{i, j}\left(1-A_{i, j}\right) & (1-3) \\
\frac{\mathrm{d} A_{i, j}}{\mathrm{~d} t}=e_{\mathrm{a}}\left(1-A_{i, j}\right) \max \left\{E_{i, j}-N_{i, j}, 0\right\} & (1-4)
\end{array}
$$

The diffusion of $E$ was calculated as follows: $d_{\mathrm{e}} \Delta E_{i, j}=d_{e}\left(E_{i+1, j}+E_{i-1, j}+E_{i, j-1}+\right.$ $\left.E_{i, j-1}-4 E_{i, j}\right) / \mathrm{d} x^{2} . k_{\mathrm{e}}, k_{\mathrm{n}}$, and $k_{\mathrm{d}}$ are passive degradation rates of EGF, N, and Dl, respectively. $a_{e}$ and $a_{\mathrm{d}}$ indicate EGF and Dl regulation by AS-C, respectively. When $A$ $=1$, the cells are fully differentiated as NBs. $e_{\mathrm{a}}$ reflects the speed of differentiation under the control of EGF and N, and is set to 100. The other parameters are set to 1 unless otherwise noted.

Linear cis-inhibition: In the previous model, the trans-activation and cisinhibition are linear (1-2). $k_{\mathrm{n}}=1, d_{\mathrm{t}}=0.25$, and $d_{\mathrm{c}}=0.1$ (Fig. 1e). In this study, (1-2) is replaced with the following.

Step function: (1-2) is replaced with (2-1) and (2-2). cis-inhibition does not occur when $D$ is below the threshold $\left(e_{c}\right)$, but quickly induces $\mathrm{N}$ inactivation when it exceeds the threshold. $k_{\mathrm{n}}=1, d_{\mathrm{t}}=0.25, d_{\mathrm{c}}=0.1$, and $e_{\mathrm{c}}=0.07$ (Figs. If and $2 \mathrm{~b}$ ). Magnitude $\left(d_{c}\right)$ and threshold $\left(e_{c}\right)$ of cis-inhibition are changed in the range $0.07-0.15$ and $0.04-0.08$, respectively (Supplementary Figure 1 ).

$$
\begin{aligned}
& \frac{\mathrm{d} N_{i, j}}{\mathrm{~d} t}=-k_{\mathrm{n}} N_{i, j}+d_{\mathrm{t}} \sum_{l, m \in \Lambda_{i, j}} D_{l, m}-d_{\mathrm{c}} H\left(D_{i, j}\right) \\
& H(x)= \begin{cases}1 & \left(x \geq e_{c}\right) \\
0 & \left(x<e_{c}\right)\end{cases}
\end{aligned}
$$

Hill function: (1-2) is replaced with (3-1). Hill's coefficient $\left(n_{\mathrm{t}}\right.$ and $\left.n_{\mathrm{c}}\right)$ and activation coefficient $\left(k_{\mathrm{t}}\right.$ and $\left.k_{\mathrm{c}}\right)$ specify the kinetics of the reaction. $k_{\mathrm{n}}=1, d_{\mathrm{t}}=$ $0.25, d_{\mathrm{c}}=0.1, n_{\mathrm{t}}=1, k_{\mathrm{t}}=1, n_{\mathrm{c}}=5$, and $k_{\mathrm{c}}=0.09$ (Figs. $1 \mathrm{~g}$ and $2 \mathrm{c}$ ). Hill's coefficient $\left(n_{\mathrm{c}}\right)$ and activation coefficient $\left(k_{\mathrm{c}}\right)$ of cis-inhibition are changed in the 
range $2-7$ and $0.07-0.11$, respectively (Supplementary Figure 2).

$$
\frac{\mathrm{d} N_{i, j}}{\mathrm{~d} t}=-k_{\mathrm{n}} N_{i, j}+\frac{d_{\mathrm{t}}\left(\sum_{l, m \in \Lambda_{i, j}} D_{l, m}\right)^{n_{\mathrm{t}}}}{k_{\mathrm{t}}^{n_{\mathrm{t}}}+\left(\sum_{l, m \in \Lambda_{i, j}} D_{l, m}\right)^{n_{\mathrm{t}}}}-\frac{d_{\mathrm{c}} D_{i, j}^{n_{\mathrm{c}}}}{k_{\mathrm{c}}^{n_{\mathrm{c}}}+D_{i, j}^{n_{\mathrm{c}}}}
$$

Five-variable model: (1-2) is replaced with (4-1) and (4-2). $F$ and $S$ represent the expression level of full-length $\mathrm{N}$ and $\mathrm{N}$ signal activity, respectively, whose initial conditions are 1 and 0 in all cells. $F_{\max }, n$, and $k_{\mathrm{t}}$ represent maximum expression level, recovery rate, and spontaneous degradation rate of full-length $\mathrm{N}$, respectively. $d_{\mathrm{t}}$ and $d_{\mathrm{c}}$ represent magnitudes of the trans-activation and cis-inhibition, respectively. Hill's coefficient $\left(n_{\mathrm{t}}\right.$ and $\left.n_{\mathrm{c}}\right)$ and activation coefficient $\left(K_{1}\right.$ and $\left.K_{2}\right)$ specify the kinetics of the reaction. $k_{\mathrm{t}}$ represents the degradation rate of full-length $\mathrm{N}$ upon trans-activation. $k_{\mathrm{f}}$ represents passive degradation rate of active form of $\mathrm{N}$. $n=1, F_{\max }=1, k_{\mathrm{f}}=0.1, d_{\mathrm{c}}=5, n_{\mathrm{t}}=1, K_{1}=0.3, k_{\mathrm{t}}=0.1, d_{\mathrm{t}}=0.25$, and $k_{\mathrm{s}}=2$. Hill's coefficient $\left(n_{\mathrm{c}}\right)$ and activation coefficient $\left(K_{2}\right)$ for Hill function in cisinhibition are changed in the range $1-7$ and $0.05-0.3$, respectively (Supplementary Figure 12).

$$
\begin{gathered}
\frac{\mathrm{d} F_{i, j}}{\mathrm{~d} t}=n\left(F_{\max }-F_{i, j}\right)-k_{\mathrm{f}} F_{i, j}-\frac{d_{\mathrm{c}} F_{i, j} D_{i, j}^{n_{\mathrm{c}}}}{K_{2}^{n_{\mathrm{c}}}+D_{i, j}^{n_{\mathrm{c}}}}-\frac{k_{\mathrm{t}} F_{i, j}\left(\sum_{l, m \in \Lambda_{i, j}} D_{l, m}\right)^{n_{\mathrm{t}}}}{K_{1}^{n_{\mathrm{t}}}+\left(\sum_{l, m \in \Lambda_{i, j}} D_{l, m}\right)^{n_{\mathrm{t}}}} \\
\frac{\mathrm{d} S_{i, j}}{\mathrm{~d} t}=\frac{d_{\mathrm{t}} F_{i, j}\left(\sum_{l, m \in \Lambda_{i, j}} D_{l, m}\right)^{n_{\mathrm{t}}}}{K_{1}^{n_{\mathrm{t}}}+\left(\sum_{l, m \in \Lambda_{i, j}} D_{l, m}\right)^{n_{\mathrm{t}}}}-k_{\mathrm{s}} S_{i, j}
\end{gathered}
$$

Two- and one-dimensional plots in Figs. 1e-g, 2b, c, and Supplementary Figures 1,2 , and 12 are the snapshots when the state of differentiation of the central cell exceeds $0.5\left(A_{13,13}>0.5\right)$. The source codes for the numerical simulations will be deposited to a public repository service.

Fly strains. Fly strains were maintained on standard Drosophila medium at $25^{\circ} \mathrm{C}$. rab4 RNAi was performed at 25 and $30^{\circ} \mathrm{C}$. The following mutant and transgenic flies were used: $D l^{R e v F 10} F R T 82 B, U A S-D l^{3-1}, U A S-D l$ RNAi (strong: BDSC36784; mild: V52188), Dl-GFP (BDSC59819), rab7-EYFP (BDSC62545L), rab4-EYFP (BDSC62542), UAS-rab7 DN (BDSC9778), UAS-rab7 RNAi (BDSC27051, VDRC40338), UAS-rab4 ${ }^{\text {DN }}$ (BDSC9768, BDSC9769), $v p s 2^{p^{p 6}}$ FRT82B, $v p s 22^{\text {NN31 }}$ FRT82B, UAS-vps2 RNAi (BDSC38995), UAS-vps 22RNAi (BDSC38289), UAS vps23 RNAi (BDSC38306), UAS-vps25 RNAi (BDSC26286), UAS-vps24 RNAi (BDSC38281), UAS-vps32 RNAi (BDSC38305), UAS- $v p s 20$ RNAi (BDSC40894), UAS- vps36 RNAi (BDSC38286), UAS-vps37B RNAi (BDSC44010), UAS-vha68-2 RNAi (BDSC34582), vha68-2 $2^{R 6}$ FRT40A (BDSC39621), UAS-rbcn3A RNAi (BDSC34612), optix-Gal4 (NP2631), Ay-Gal450, UAS-CD8GFP, UAS-GFP, hs-flp, $u b i-G F P$ FRT82B, and $u b i-R F P$ FRT82B. $\mathrm{N}$ activity was visualized by $E(s p l)-m \gamma G F P$ $(m \gamma G F P)$ and NRE-dVenus ${ }^{32,33}$.

Clonal analysis. $D l$ overexpression clones were generated by crossing $h s-f l p ; A y$ Gal4 UAS-GFP strain with UAS-D $l^{3-1}$ and applying 15 min heat shock at $34^{\circ} \mathrm{C} . \mathrm{Dl}$ mutant clones were generated by crossing $h s$-flp; ubi-GFP FRT82B strain with $D l^{R e v F 10} F R T 82 B$ and applying 50 min heat shock at $37^{\circ} \mathrm{C}$. vps mutant clones were generated by crossing $h s-f l p ; m \gamma G F P ; u b i-R F P$ FRT82B or hs-flp; NRE-dVenus; ubiRFP FRT82B with vps FRT82B and applying 50 min heat shock at $37^{\circ} \mathrm{C}$. run-Gal4 MARCM clones were generated by crossing run-Gal4 UAS-CD8GFP FRT19F; FRT40A strain with $h s$-flp; tubP-Gal80 FRT40A and applying 15 min heat shock at $34^{\circ} \mathrm{C}$

Generation of run-Gal4 strain. The second exon containing the translational start site of run was replaced by the fragment containing attP site and GMR-white via the homologous recombination with the homology arms for run (runLp and runRp) ${ }^{51}$ in the presence of FRT19F (Kyoto125719). The Gal4 containing fragment was integrated into the attP site in the run locus by co-injecting PhiC31 plasmid and run-Gal4/pGEattB, which contains attB site, the fragment containing the translational start site of run (run5pri) and GMR-white. The expression pattern of run-Gal4 was verified by expressing UAS-GFP and co-staining with anti-Run antibody. runLp (2.0 kb), runRp (2.2 kb, and run5pri $(153 \mathrm{bp})$ were amplified using the following PCR primers: runLp5_NotI (catgcggecgcccaagtatgacacttccgcatc), runLp3_KpnI (catggtaccctttatcgggggtcacttggaa), runRp5_AscI (catggcgcgcccgggagccaagaagtaagcaaa), runRp3_XhoI (catctcgagggccaactgtgataggaagttc), runGal5Not (catgcggccgcttccaagtgacccccgataaag), and runGal3-Bam (catggatcctgtgttgttggccaccatcgtt). Underlined sequences are homologous to the genomic sequences.

Histochemistry. Immunohistochemistry was performed as described below ${ }^{48}$. Details are available upon request. Brains were dissected in phosphate-buffered saline (PBS), transferred to ice-cold $0.8 \%$ formaldehyde/PBS solution, and fixed in $4 \%$ formaldehyde/PBS at room temperature for 30-60 min. The brains were washed in PBT ( $0.3 \%$ Triton X in PBS) and blocked in 5-10\% normal serum/PBT solution at room temperature for 30-60 min. Primary antibody reaction was performed in a solution containing primary antibodies and $1 \%$ normal serum in PBT at $4{ }^{\circ} \mathrm{C}$ overnight. The brains were washed in PBT. Secondary antibody reaction was performed in a solution containing secondary antibodies and $1 \%$ normal serum in PBT at $4{ }^{\circ} \mathrm{C}$ overnight. After washing in PBT and PBS, the brains were mounted in VECTASHIELD (Vector Laboratories).

The following primary antibodies were used: guinea pig anti-Lsc (1:1200), mouse anti-Dl (1:20; DSHB C594.9B), mouse anti-N (1:20; DSHB C17.9C6), rat anti-Ecad (1:20; DSHB DCAD2), rabbit anti-Klu (1:1000; Xiaohang Yang, Singapore), guinea pig anti-Run (1:500; Asian Distribution Center for Segmentation Antibodies, Mishima, Japan), and rabbit anti-Rab7 (1:600; Akira Nakamura, Kumamoto University, Japan). The following secondary antibodies were used: anti-mouse Cy3 (1:200; Jackson ImmunoResearch 715-165-151), antimouse Cy5 (1:200; Jackson ImmunoResearch 715-175-151), anti-guinea pig Alexa647 (1:200; Jackson ImmunoResearch 712-605-150), and anti-rabbit Alexa546 (1:200; Invitrogen A-11035). Confocal images were obtained by Zeiss LSM880 and processed using ZEN, Fiji, and Adobe Photoshop. Signal intensity was quantified within the indicated rectangle areas by ImageJ.

In situ hybridization. In situ hybridization was performed as briefly described below. Details are available upon request. Larval brains were dissected in ice-cold PBS, transferred to $4 \%$ formaldehyde/PBS solution, and fixed at $4{ }^{\circ} \mathrm{C}$ overnight. The formaldehyde solution was removed, and the brains were washed with PBS and $70 \%$ ethanol. Incubation at room temperature for 2-5 min after replacing the solution with Wash Buffer A (100 $\mu$ l Stellaris RNA FISH Wash Buffer A, $300 \mu \mathrm{l}$ nuclease-free water, $50 \mu$ deionized formamide). Replacing the solution with Hybridization Buffer ( $90 \mu$ l Hybridization Buffer mix with $2 \mu$ l Stellaris RNA FISH probe designed for exon 6 of $N$ gene labeled with Quasar 570), the brains were incubated at $37^{\circ} \mathrm{C}$ for $8 \mathrm{~h}$ in the dark. Incubation at $37^{\circ} \mathrm{C}$ for $30 \mathrm{~min}$ after replacing the solution with Wash Buffer A. Replacing the solution with TO-PRO3/PBS, the brains were incubated at $37^{\circ} \mathrm{C}$ for $30 \mathrm{~min}$. After washing in PBS, the brains were mounted in $80 \%$ glycerol in PBS.

Duolink PLA. PLA was performed using Duolink (Sigma-Aldrich). The dissected fly brains were fixed in $4 \%$ formaldehyde in PBT ( $0.3 \%$ Triton X in PBS). After finishing the Lsc immunostaining, the brains were incubated with Duolink blocking solution for $60 \mathrm{~min}$ at $37^{\circ} \mathrm{C}$ and in the Duolink antibody diluent containing mouse anti- $\mathrm{Dl}$ and rabbit anti-Rab7 antibodies for overnight at $4{ }^{\circ} \mathrm{C}$. The brains were washed in Duolink Wash Buffer A three times at room temperature, incubated in a solution containing PLA PLUS anti-mouse and PLA MINUS antirabbit probes for $120 \mathrm{~min}$ at $37^{\circ} \mathrm{C}$, washed in Duolink Wash Buffer A, and incubated with Duolink ligation stock for $60 \mathrm{~min}$ at $37^{\circ} \mathrm{C}$. Then, the brains were washed in Wash Buffer A and incubated with Duolink polymerase in Duolink amplification stock for $100 \mathrm{~min}$ at $37^{\circ} \mathrm{C}$. Finally, the brains were washed in Wash Buffer B and mounted in Duolink in situ mounting medium with DAPI $\left(4^{\prime}, 6-\right.$ diamidino-2-phenylindole).

LysoTracker staining. Fly brains were dissected in cold S2 medium and incubated in PBS containing LysoTrancker Red DND99 (1:1000, Thermo Fisher) for 30 min at room temperature. The brains were washed in PBS and fixed in $4 \%$ formaldehyde in PBS. After washing in PBS, the brains were mounted in VECTASHIELD (Vector Laboratories)

Statistics and reproducibility. For quantification and statistical analysis, distinct brain areas or samples were measured and analyzed as indicated below. Two-sided $t$ test was used for the statistical test. Image intensities were not artificially processed, except as otherwise noted. When statistics were not applicable, experiments were independently repeated at least three times with similar results.

Image quantification. Signal intensity was quantified within the indicated rectangle areas using Fiji (Figs. 1c-d, 2e-i, 4, 5, and 6f-n). Coloc 2 function of Fiji was used to calculate the Pearson's $R$ value to quantify the colocalization between two different signals (Figs. 3i, $\mathrm{k}$ and $6 \mathrm{~b}, \mathrm{o}$ ).

The $x y$ coordinates of cells were obtained by manually selecting individual cells expressing Run or Bsh (Fig. $7 \mathrm{~g}, \mathrm{~h}$ ). The $x$ coordinate of the origin was calculated as the average of cell locations along the $x$-axis. The $y$ coordinate of the origin was determined so that the standard deviation of $R$, the distance from the origin to cells, is minimized. $\Delta \theta$, the angle of mutant area from the origin, was determined based on the mutant area of Bsh-positive cells. The same $\Delta \theta$ was used to determine the control area to be analyzed at the dorsal-ventral boundary where optix-Gal4 is not expressed. Sizes of the intersection between Run- and Bsh-positive areas in the control and mutant areas were calculated using "convhull," "polyshape," and "intersects" functions of MATLAB (Fig. 7i).

Ethical approval. We have complied with all relevant ethical regulations for animal testing and research. This study did not require an ethical approval. 
Reporting summary. Further information on research design is available in the Nature Research Reporting Summary linked to this article.

\section{Data availability}

The authors declare that the data supporting the findings of this study are available within the paper, or available upon request. Source data are provided with this paper.

\section{Code availability}

The source codes for the numerical simulations and image quantifications are deposited in a public repository service (https://github.com/satouma7/TwinPeaksCode).

Received: 17 February 2020; Accepted: 16 March 2021; Published online: 07 April 2021

\section{References}

1. Artavanis-Tsakonas, S., Rand, M. D. \& Lake, R. J. Notch signaling: cell fate control and signal integration in development. Science 284, 770-776 (1999).

2. Simpson, P. Lateral inhibition and the development of the sensory bristles of the adult peripheral nervous system of Drosophila. Development 109, 509-519 (1990).

3. Sato, M., Yasugi, T., Minami, Y., Miura, T. \& Nagayama, M. Notch-mediated lateral inhibition regulates proneural wave propagation when combined with EGF-mediated reaction diffusion. Proc. Natl Acad. Sci. USA 113, E5153-E5162 (2016).

4. Tanaka, Y., Yasugi, T., Nagayama, M., Sato, M. \& Ei, S. I. JAK/STAT guarantees robust neural stem cell differentiation by shutting off biological noise. Sci. Rep. 8, 12484 (2018).

5. Kunisch, M., Haenlin, M. \& Campos-Ortega, J. A. Lateral inhibition mediated by the Drosophila neurogenic gene delta is enhanced by proneural proteins. Proc. Natl Acad. Sci. USA 91, 10139-10143 (1994).

6. Bray, S. \& Furriols, M. Notch pathway: making sense of suppressor of hairless. Curr. Biol. 11, R217-R221 (2001).

7. Struhl, G. \& Adachi, A. Nuclear access and action of notch in vivo. Cell 93, 649-660 (1998).

8. Yuan, Z. et al. Structure and function of the $\mathrm{Su}(\mathrm{H})$-Hairless Repressor complex, the major antagonist of notch signaling in Drosophila melanogaster. PLoS Biol. 14, e1002509 (2016).

9. del Alamo, D. \& Schweisguth, F. Notch signalling: receptor cis-inhibition to achieve directionality. Curr. Biol. 19, R683-R684 (2009).

10. Miller, A. C., Lyons, E. L. \& Herman, T. G. cis-Inhibition of Notch by endogenous Delta biases the outcome of lateral inhibition. Curr. Biol. 19, 1378-1383 (2009).

11. Sprinzak, D. et al. Cis-interactions between Notch and Delta generate mutually exclusive signalling states. Nature 465, 86-90 (2010).

12. Berndt, N. et al. Ubiquitylation-independent activation of Notch signalling by Delta. eLife 6, https://doi.org/10.7554/eLife.27346 (2017).

13. del Alamo, D., Rouault, H. \& Schweisguth, F. Mechanism and significance of cis-inhibition in Notch signalling. Curr. Biol. 21, R40-R47 (2011).

14. Sakamoto, K., Ohara, O., Takagi, M., Takeda, S. \& Katsube, K. Intracellular cell-autonomous association of Notch and its ligands: a novel mechanism of Notch signal modification. Dev. Biol. 241, 313-326 (2002).

15. Moberg, K. H., Schelble, S., Burdick, S. K. \& Hariharan, I. K. Mutations in erupted, the Drosophila ortholog of mammalian tumor susceptibility gene 101, elicit non-cell-autonomous overgrowth. Dev. Cell 9, 699-710 (2005).

16. Thompson, B. J. et al. Tumor suppressor properties of the ESCRT-II complex component Vps25 in Drosophila. Dev. Cell 9, 711-720 (2005).

17. Vaccari, T. \& Bilder, D. The Drosophila tumor suppressor vps 25 prevents nonautonomous overproliferation by regulating notch trafficking. Dev. Cell $\mathbf{9}$, 687-698 (2005).

18. Herz, H. M. et al. vps 25 mosaics display non-autonomous cell survival and overgrowth, and autonomous apoptosis. Development 133, 1871-1880 (2006).

19. Vaccari, T., Lu, H., Kanwar, R., Fortini, M. E. \& Bilder, D. Endosomal entry regulates Notch receptor activation in Drosophila melanogaster. J. Cell Biol. 180, 755-762 (2008).

20. Aoyama, N. et al. Loss- and gain-of-function analyses of vacuolar protein sorting 2 in Notch signaling of Drosophila melanogaster. Genes Genet. Syst. 88, 45-57 (2013).

21. Fortini, M. E. \& Bilder, D. Endocytic regulation of Notch signaling. Curr. Opin. Genet Dev. 19, 323-328 (2009).

22. Vaccari, T. et al. Comparative analysis of ESCRT-I, ESCRT-II and ESCRT-III function in Drosophila by efficient isolation of ESCRT mutants. J. Cell Sci. 122, 2413-2423 (2009).
23. Jaekel, R. \& Klein, T. The Drosophila Notch inhibitor and tumor suppressor gene lethal (2) giant discs encodes a conserved regulator of endosomal trafficking. Dev. Cell 11, 655-669 (2006).

24. Schneider, M., Troost, T., Grawe, F., Martinez-Arias, A. \& Klein, T. Activation of Notch in lgd mutant cells requires the fusion of late endosomes with the lysosome. J. Cell Sci. 126, 645-656 (2013).

25. Egger, B., Boone, J. Q., Stevens, N. R., Brand, A. H. \& Doe, C. Q. Regulation of spindle orientation and neural stem cell fate in the Drosophila optic lobe. Neural Dev. 2, 1 (2007).

26. Yasugi, T., Umetsu, D., Murakami, S., Sato, M. \& Tabata, T. Drosophila optic lobe neuroblasts triggered by a wave of proneural gene expression that is negatively regulated by JAK/STAT. Development 135, 1471-1480 (2008).

27. Yasugi, T., Sugie, A., Umetsu, D. \& Tabata, T. Coordinated sequential action of EGFR and Notch signaling pathways regulates proneural wave progression in the Drosophila optic lobe. Development 137, 3193-3203 (2010).

28. Reddy, B. V., Rauskolb, C. \& Irvine, K. D. Influence of fat-hippo and notch signaling on the proliferation and differentiation of Drosophila optic neuroepithelia. Development 137, 2397-2408 (2010).

29. Contreras, E. G., Egger, B., Gold, K. S. \& Brand, A. H. Dynamic Notch signalling regulates neural stem cell state progression in the Drosophila optic lobe. Neural Dev. 13, 25 (2018).

30. Li, X. et al. Temporal patterning of Drosophila medulla neuroblasts controls neural fates. Nature 498, 456-462 (2013).

31. Suzuki, T., Kaido, M., Takayama, R. \& Sato, M. A temporal mechanism that produces neuronal diversity in the Drosophila visual center. Dev. Biol. 380, 12-24 (2013).

32. Almeida, M. S. \& Bray, S. J. Regulation of post-embryonic neuroblasts by Drosophila Grainyhead. Mech. Dev. 122, 1282-1293 (2005).

33. Housden, B. E., Millen, K. \& Bray, S. J. Drosophila reporter vectors compatible with PhiC31 integrase transgenesis techniques and their use to generate new notch reporter fly lines. G3 2, 79-82 (2012).

34. Fehon, R. G. et al. Molecular interactions between the protein products of the neurogenic loci Notch and Delta, two EGF-homologous genes in Drosophila. Cell 61, 523-534 (1990).

35. Glittenberg, M., Pitsouli, C., Garvey, C., Delidakis, C. \& Bray, S. Role of conserved intracellular motifs in Serrate signalling, cis-inhibition and endocytosis. EMBO J. 25, 4697-4706 (2006).

36. Dunst, S. et al. Endogenously tagged rab proteins: a resource to study membrane trafficking in Drosophila. Dev. Cell 33, 351-365 (2015).

37. Tanaka, T. \& Nakamura, A. The endocytic pathway acts downstream of Oskar in Drosophila germ plasm assembly. Development 135, 1107-1117 (2008).

38. Vanlandingham, P. A. \& Ceresa, B. P. Rab7 regulates late endocytic trafficking downstream of multivesicular body biogenesis and cargo sequestration. J. Biol. Chem. 284, 12110-12124 (2009).

39. Mohrmann, K., Gerez, L., Oorschot, V., Klumperman, J. \& van der Sluijs, P. Rab4 function in membrane recycling from early endosomes depends on a membrane to cytoplasm cycle. J. Biol. Chem. 277, 32029-32035 (2002).

40. Jorg, D. J. et al. The proneural wave in the Drosophila optic lobe is driven by an excitable reaction-diffusion mechanism. eLife 8, https://doi.org/10.7554/ eLife.40919 (2019).

41. $\mathrm{Li}, \mathrm{B}$. et al. The retromer complex safeguards against neural progenitorderived tumorigenesis by regulating Notch receptor trafficking. eLife 7, https:// doi.org/10.7554/eLife.38181 (2018).

42. Couturier, L., Trylinski, M., Mazouni, K., Darnet, L. \& Schweisguth, F. A fluorescent tagging approach in Drosophila reveals late endosomal trafficking of Notch and Sanpodo. J. Cell Biol. 207, 351-363 (2014).

43. Yan, Y., Denef, N. \& Schupbach, T. The vacuolar proton pump, V-ATPase, is required for notch signaling and endosomal trafficking in Drosophila. Dev. Cell 17, 387-402 (2009).

44. Wissel, S. et al. Time-resolved transcriptomics in neural stem cells identifies a v-ATPase/Notch regulatory loop. J. Cell Biol. 217, 3285-3300 (2018).

45. Vaccari, T., Duchi, S., Cortese, K., Tacchetti, C. \& Bilder, D. The vacuolar ATPase is required for physiological as well as pathological activation of the Notch receptor. Development 137, 1825-1832 (2010).

46. Yang, X., Bahri, S., Klein, T. \& Chia, W. Klumpfuss, a putative Drosophila zinc finger transcription factor, acts to differentiate between the identities of two secondary precursor cells within one neuroblast lineage. Genes Dev. 11, 1396-1408 (1997).

47. Hasegawa, E. et al. Concentric zones, cell migration and neuronal circuits in the Drosophila visual center. Development 138, 983-993 (2011).

48. Hasegawa, E., Kaido, M., Takayama, R. \& Sato, M. Brain-specific-homeobox is required for the specification of neuronal types in the Drosophila optic lobe. Dev. Biol. 377, 90-99 (2013).

49. Imayoshi, I. et al. Oscillatory control of factors determining multipotency and fate in mouse neural progenitors. Science 342, 1203-1208 (2013).

50. Ito, K., Awano, W., Suzuki, K., Hiromi, Y. \& Yamamoto, D. The Drosophila mushroom body is a quadruple structure of clonal units each of which 
contains a virtually identical set of neurones and glial cells. Development 124, 761-771 (1997).

51. Huang, J., Zhou, W., Dong, W., Watson, A. M. \& Hong, Y. From the cover: directed, efficient, and versatile modifications of the Drosophila genome by genomic engineering. Proc. Natl Acad. Sci. USA 106, 8284-8289 (2009).

\section{Acknowledgements}

We thank members of Sato lab for supporting fly work, T. Kawauchi, K. Matsuno for helpful discussion, S. Bray, A. Nakamura, J. Skeath, Bloomington Drosophila Stock

Center, Vienna Drosophila Resource Center, Drosophila Genetic Resource Center, Kyoto, Asian Distribution Center for Segmentation Antibodies, Mishima, and Developmental Studies Hybridoma Bank for flies and antibodies. This work was supported by CREST from JST (JPMJCR14D3 to M.S., S.-I.E. and JPMJCR15D2 to M.N.), Grant-in-Aid for Scientific Research (B), (C), Grant-in-Aid for Scientific Research on Innovative Areas and Grant-in-Aid for Early-Career Scientists from MEXT (17H03542, 17H05739, 17H05761, and 19H04771 to M.S., 19K06674, 19H04956, and 20H05030 to T.Y., and JP20K14364 to Y.T.), Takeda Science Foundation (to M.S. and T.Y.), Cooperative Research of "Network Joint Research Center for Materials and Devices" (to M.S.)

\section{Author contributions}

M.W. and M.S. conceived and designed the experiments. X.H., C.L., R.T., T.Y., and M.S. performed experiments. M.W. and M.S. acquired, analyzed, and interpreted the data. S.-I. E., M.N., Y.T., and M.S. formulated the mathematical models. M.W. and M.S. wrote the manuscript.

\section{Competing interests}

The authors declare no competing interests.

\section{Additional information}

Supplementary information The online version contains supplementary material available at https://doi.org/10.1038/s41467-021-22442-3.

Correspondence and requests for materials should be addressed to M.S.

Peer review information Nature Communications thanks David Sprinzak, Thomas Vaccari and the other, anonymous, reviewer(s) for their contribution to the peer review of this work. Peer reviewer reports are available.

Reprints and permission information is available at http://www.nature.com/reprints

Publisher's note Springer Nature remains neutral with regard to jurisdictional claims in published maps and institutional affiliations.

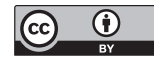

Open Access This article is licensed under a Creative Commons Attribution 4.0 International License, which permits use, sharing, adaptation, distribution and reproduction in any medium or format, as long as you give appropriate credit to the original author(s) and the source, provide a link to the Creative Commons license, and indicate if changes were made. The images or other third party material in this article are included in the article's Creative Commons license, unless indicated otherwise in a credit line to the material. If material is not included in the article's Creative Commons license and your intended use is not permitted by statutory regulation or exceeds the permitted use, you will need to obtain permission directly from the copyright holder. To view a copy of this license, visit http://creativecommons.org/ licenses/by/4.0/.

(C) The Author(s) 2021 